\title{
The deubiquitinase USP10 regulates KLF4 stability and suppresses lung tumorigenesis
}

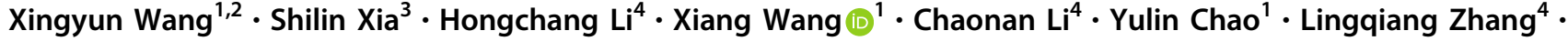 \\ Chuanchun $\operatorname{Han}^{1}$
}

Received: 24 July 2019 / Revised: 4 November 2019 / Accepted: 6 November 2019 / Published online: 20 November 2019

(c) The Author(s), under exclusive licence to ADMC Associazione Differenziamento e Morte Cellulare 2019

\begin{abstract}
Krüppel-like factor 4 (KLF4), a key transcription factor, acts as a multifunctional player involved in the progression of numerous aggressive cancers. The proteasome-dependent pathway is one of the main modalities in controlling KLF4 abundance at a posttranslational level. Although some of the ubiquitin ligases have been identified, the deubiquitinases of KLF4 and the regulatory function remain unexplored. Here, by screening ubiquitin-specific proteases that may interact with KLF4, we found ubiquitin-specific peptidase 10 (USP10) as a deubiquitinating enzyme for KLF4. Forced expression of USP10 remarkably increases KLF4 protein level by blocking the latter degradation, whereas the depletion of USP10 promotes KLF4 degradation and thus enhances tumorigenesis. Loss of USP10 in mice downregulates KLF4 expression and accelerates $\mathrm{Kras}^{\mathrm{G} 12 \mathrm{D}}$-driven lung adenocarcinoma initiation and progression. In addition, our data revealed that KLF4 can facilitate the transcription of tumor suppressor TIMP3 by directly binding to the TIMP3 promoter. Clinically, reduction of USP10 expression, concomitant with decreased KLF4 and TIMP3 abundance in carcinoma tissue, predicts poor prognosis of lung cancer patient. Taken together, our results demonstrate that USP10 is a critical regulator of KLF4, pinpointing USP10KLF4-TIMP3 axis as a promising therapeutic target in lung cancer.
\end{abstract}

These authors contributed equally: Xingyun Wang, Shilin Xia, Hongchang Li, Xiang Wang

Edited by: V. D'Angiolella

Supplementary information The online version of this article (https:// doi.org/10.1038/s41418-019-0458-7) contains supplementary material, which is available to authorized users.

Lingqiang Zhang

zhanglq@ nic.bmi.ac.cn

$\triangle$ Chuanchun Han

hanchuanchun@163.com

1 Institute of Cancer Stem Cell, Dalian Medical University, Dalian 116044, China

2 Women's Hospital of Nanjing Medical University, Nanjing Maternity and Child Health Care Hospital, Nanjing 210004, China

3 Clinical Laboratory of Integrative Medicine, The First Affiliated Hospital of Dalian Medical University, Dalian 116027, China

4 State Key Laboratory of Proteomics, National Center for Protein Sciences (Beijing), Beijing Institute of Lifeomics, 100850 Beijing, China

\section{Introduction}

Lung cancer is the leading cause of cancer-related death worldwide with high mortality and poor prognosis [1]. Nonsmall cell lung cancer (NSCLC) accounts for $80 \%$ of all lung cancer cases and is further divided into three categories including adenocarcinoma (ADC), squamous cell carcinoma, and large cell carcinoma. Mutations in multiple genetic pathways, including EGFR, TP53, and K-RAS, contribute to lung tumorigenesis [2-4]. Ubiquitination is a highly regulated, reversible posttranslational protein modification that serves to target proteins for degradation or regulate protein function by the ubiquitin (Ub) system [5]. A large number of studies have demonstrated that ubiquitination plays an essential role in cancer pathogenesis and revealed the great therapeutic potential of targeting ubiquitination in multiple cancers including lung cancer [6-10].

Like other posttranslational modifications, ubiquitination can be reversed by deubiquitinases (DUBs), which could cleave and remove the $\mathrm{Ub}$ chains from substrate proteins [11]. In human proteome, 100 DUBs have been found, which can be divided into six subfamilies: USPs, OTUs, 
JAMMs, MJDs MINDYs, and UCHs [12, 13]. Accumulating evidence has demonstrated that the deregulation of the deubiquitination process frequently occurs in tumorigenesis and consequently many proteins are affected by this process $[14,15]$. For example, the tumor suppressor PTEN is de-polyubiquitinated by USP13 and OTUD3, which suppress PTEN degradation and breast tumorigenesis, or is de-monoubiquitinated by USP7 that modulates its subcellular localization [16-18]. c-Myc is directly depolyubiquitinated by USP22, USP28, and USP37, which can increase c-Myc stability and tumorigenesis, or is indirectly reduced by USP9X through stabilizing its E3 ligase Fbw7 that inhibits colorectal cancer formation [19-22].

KLF4, a member of the KLF-like factor subfamily of zinc finger proteins, has an essential role in cell-fate decisions, including DNA damage response, inflammation, apoptosis and stem cell reprogramming [23-26]. Dysfunction of KLF4 has been observed in a number of human diseases, especially in cancer [27]. Interestingly, substantial evidence reveal an ambivalent nature for KLF4 in tumorigenesis as either a tumor suppressor or an oncogene in a tissue-specific manner [25, 28, 29]. In lung cancer, KLF4 is believed to function as a tumor suppressor and inhibits lung tumorigenesis by its transactivation of cell cycle inhibitor $\mathrm{p} 21^{\mathrm{Cip} 1 / \mathrm{Waf} 1}$ and suppression of several cell cycle promoting genes [24, 28].

Emerging evidence revealed that KLF4 is unstable and regulated by ubiquitination [30]. The E3 ligase $\beta$-TrCP, pVHL, FBXO32, and Mule are reported to promote KLF4 ubiquitination and degradation [31-34]. $\beta$-TrCP ubiquitinates KLF4 in response to phosphorylation by ERK1/2 and regulates mouse embryonic stem cell renewal [32]. Tumor suppressors pVHL and FBXO32 can enhance KLF4 ubiquitination and degradation in breast cancer [33, 35]. In addition, the E3 ligase Mule controls T-cell proliferation by mediating KLF4 ubiqutination and degradation [34]. Although numerous E3 ligases have been found to promote KLF4 polyubiquitination, the DUBs for KLF4 are still undefined.

Here, we identify USP10 as a potent DUB for KLF4 de-polyubiquitination. Increased USP10 expression suppresses KLF4 degradation, leading to the decrease of lung cancer cell growth and migration. Moreover, we found that the depletion of USP10 in mice downregulates KLF4 expression, accelerates lung tumor formation, which thus results in shorted survival. Further studies reveal that the tissue inhibitor of metalloproteinases-3 (TIMP3) is a downstream target gene of KLF4 and USP10 inhibits lung tumorigenesis through activating KLF4-TIMP3 pathway. Overall, our data indicate that USP10 is a novel deubiquitinase for KLF4 and governs KLF4 stability in lung cancer.

\section{Results}

\section{USP10 enhances KLF4 expression}

To screen the potential DUBs that may regulate KLF4 stability, we expressed various DUBs together with GFP-KLF4 in HEK293T cell line and determined the DUBs interacting with KLF4. We found that four DUBs including USP4, USP6, USP7, and USP10 could interact with KLF4 (Supplementary Fig. 1A-C). Subsequent experiments revealed that KLF4 protein levels were dramatically increased by the co-expression of USP10, rather than other USPs such as USP4, USP5, USP6, and USP7 in HEK293T cells (Supplementary Fig. 1D). To further confirm this, we overexpressed USP10 in lung cancer cells and found that the ectopic expression of USP10 resulted in KLF4 elevation in a dose-dependent manner (Fig. 1a and Supplementary Fig. 1E). On the contrary, the knockdown (KD) of USP10 decreased KLF4 protein levels in lung cancer cells and human bronchial epithelia cells (HBE) (Fig. 1b-d). Next, we examined whether the increase in KLF4 protein levels is dependent on the deubiquitinating enzymatic activity of USP10. Wild-type USP10 or a catalytically inactive mutant USP10 CA was overexpressed in H1299 and A549 cells. As shown in Fig. 1e, f, the wild-type USP10 instead of the CA mutant increased KLF4 protein level. These results were also confirmed in USP10 knockout (KO) lung cancer cells that were established using the CRISPR/Cas9 system. We found that ectopic expression of the wild-type USP10 not the CA mutant reversed the reduction of KLF4 by USP10 KO (Fig. 1g and Supplementary Fig. 1F).

To further investigate the effects of USP10 on KLF4 expression in vivo, Loxp-Cre strategy mediated global deletion of the Usp10 mice were introduced (Supplementary Fig. 1G, H). Consistent with the previous report, Usp $10^{-1-}$ mice died in one day after born [36]. To assess the expression of KLF4 in Usp10 KO mice, we dissected and collected various tissues from Usp $10 \mathrm{WT}$ and $K O$ mice when the mice were born. We found that the deletion of USP10 in mice significantly decreased the KLF4 expression in lung, muscle, and spleen tissues (Fig. 1h-k). Similarly, KLF4 protein levels rather than the mRNA levels were downregulated in the MEF cells from Usp10 $K O$ mice (Fig. 11 and Supplementary Fig. 1I). In addition, adeno-associated virus expressing USP10-shRNA was delivered into mouse lung by intratracheal injection for knocking down USP10 expression. The western blotting results showed that the KD of USP10 in mouse lung resulted in a remarkable decrease of KLF4 protein expression (Fig. 1m). Collectively, these results indicate that USP10 specifically increases KLF4 protein levels in vivo and in vitro. 

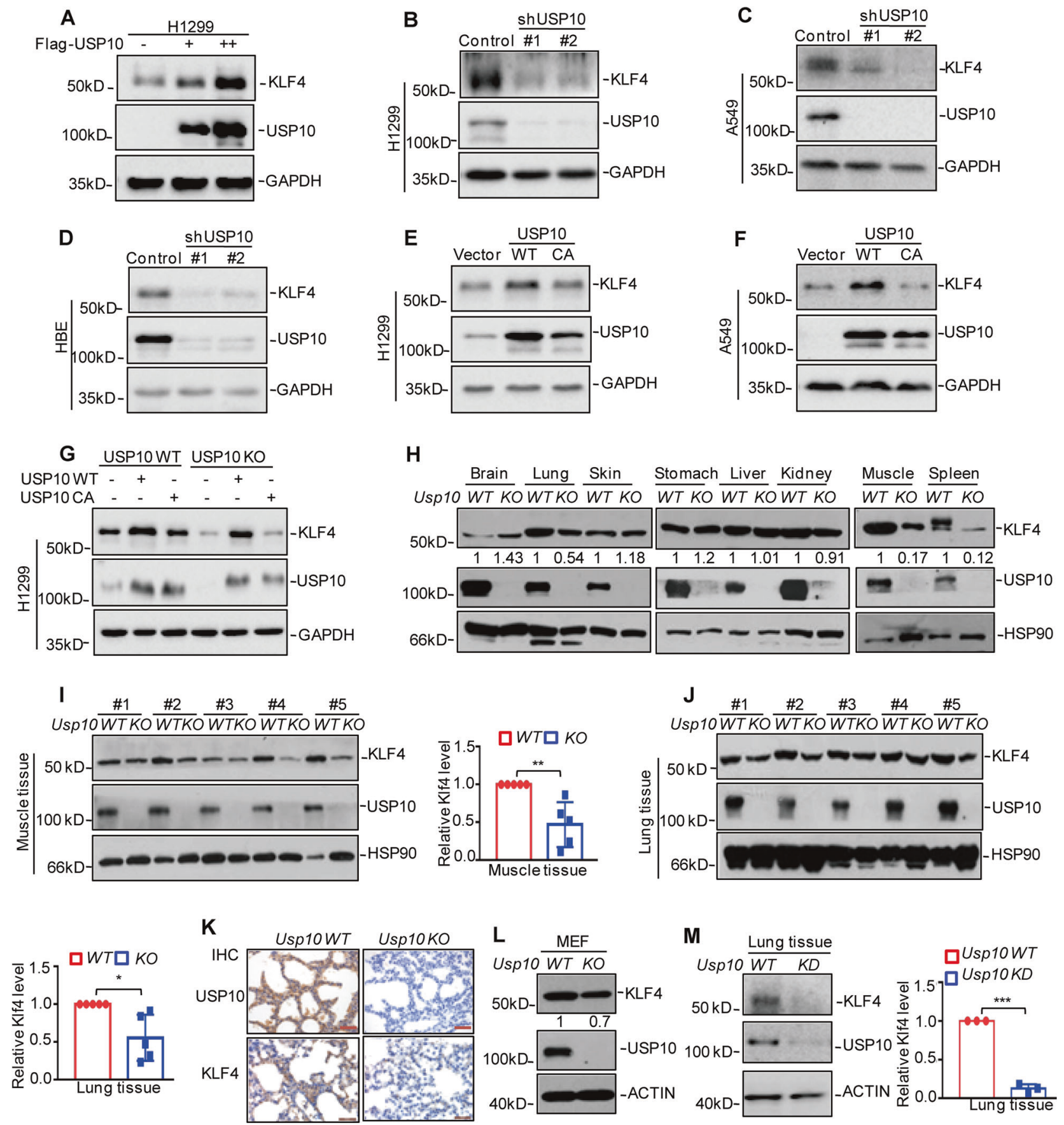

Fig. 1 USP10 enhances KLF4 expression. a Increasing amounts of USP10 were transfected into H1299 cells and KLF4 expression was detected. b-d USP10 was knocked down in H1299, A549, and HBE cells using two independent shRNAs. The protein levels of KLF4 were analyzed by western blotting. e, f USP10 WT or CA mutant was overexpressed in H1299 and A549 cells. The protein levels of KLF4 were analyzed. $\mathbf{g}$ USP10 WT or CA mutant was transfected into H1299 cells with or without endogenous USP10 knockout. The protein levels of KLF4 were analyzed. h Immunoblotting analysis protein levels of KLF4 and USP10 in different tissues from Usp10 WT and KO littlemates. i, j The protein levels of KLF4 and USP10 were analyzed by western blotting in muscle and lung tissues of Usp10 WT or $K O$ mice $(n=5)$. k Representative images from immunohistochemical staining of USP10 and KLF4 in lung tissues from Usp10 WT or KO mice. Scale bars: $50 \mu \mathrm{m}$. 1 The expression levels of KLF4 in Usp10 WT and KO MEFs were detected by western blotting. m USP10 was knocked down in adult mouse lung tissue using AAV-shRNA Control (Usp10 WT) or shRNA USP10 (Usp10 KD). After 4 weeks of infection $\left(1 \times 10^{10} \mathrm{pfu}\right)$, the expression levels of KLF4 and USP10 were analyzed by western blotting in mouse lung tissue $(n=3)$. For all panels, data are representative results of three independent experiments. $\mathbf{i}, \mathbf{j}, \mathbf{m}$ Quantification of KLF4 levels relative to HSP90 or ACTIN is shown. Results are shown as mean \pm s.d.; two-tailed Student's $t$-test, ${ }^{*} p<0.05, * * p<0.01, * * * p<0.001$. WT wild type, KO knockout, KD knockdown, CA CA mutant, IHC Immunohistochemical staining 


\section{USP10 maintains KLF4 stability and deubiquitinates KLF4}

To further validate whether USP10 affects KLF4 expression in a proteasome-dependent manner, H1299 and A549 cells with or without USP10 KD were treated by the proteasome inhibitor MG132. We found that MG132 reversed the downregulation of KLF4 although USP10 depleted (Fig. 2a and Supplementary Fig. 2A). Subsequently, we treated indicated cells with the protein synthesis inhibitor cycloheximide (CHX). Notably, the overexpression of wild-type USP10 rather than a mutant can lead to a prominent increase in the stability of endogenous KLF4 protein (Fig. 2b and Supplementary Fig. 2B). In contrast, USP10 depletion results in the destabilization of KLF4 protein (Fig. 2c, d and Supplementary Fig. 2C, D).

Considering USP10 could act as a deubiquitinase to remove ubiquitination from its substrates, we reasoned that USP10 should module KLF4 expression through deubiquitination. As expected, ectopic expression of wild-type USP10 instead of the CA mutant reduced the polyubiquitination of KLF4 (Fig. 2e, f and Supplementary Fig. 2E), whereas USP10 loss increased KLF4 polyubiquitination, which could be reversed by rescue experiments with introduction of wild-type USP10 (Fig. $2 \mathrm{~g}$, h and Supplementary Fig. 2F-H). To further verify our findings, in vitro deubiquitylation assay was performed. Consistently, the wild-type USP10 not the CA mutant removed Ub chains on KLF4 in vitro (Fig. 2i). Thus, these data suggest that USP10 governs KLF4 stability and inhibits its polyubiquitination.

\section{USP10 interacts with KLF4}

Consequent upon findings mentioned, we next determine whether USP10 directly interacts with KLF4 and is a bona fide interacting protein for KLF4. Consistent with the interaction observed in the initial screen, coimmunoprecipitation assays confirmed that ectopically expressed GFP-tagged KLF4 could be detected in FLAGtagged USP10 and vice versa (Fig. 3a, b). Moreover, purified GST-KLF4, rather than the GST control, was able to bind to FLAG-tagged USP10 under cell-free conditions, indicating a direct interaction between USP10 and KLF4 (Fig. 3c). More importantly, an interaction between endogenous USP10 and KLF4 was also detected in H1299 and A549 cells (Fig. 3d-g). Immunofluorescence assays show that USP10 and KLF4 co-localize in H1299 and A549 cells (Fig. 3h). KLF4 functions as a versatile transcription factor that up- or down-regulates its responsive genes through an activation domain or a repressive domain located near its amino terminus. The carboxyl terminus of KLF4 contains three zinc-fingers and two nuclear localization signals that mediate DNA binding and regulate subcellular localization. To map the USP10-binding region on KLF4, we expressed Flag-tagged USP10 along with different domains of KLF4 in HEK293T cells. Co-immunoprecipitation assays demonstrate that the repression domain of KLF4 is essential for its physical interaction with USP10. In particular, the amino-terminal region (aa1-aa100) of USP10 mediates the physical interaction with KLF4 (Fig. 3i-k). The aminoterminal deletion mutant of USP10 lost its effect on regulating KLF4 protein levels and ubiquitination (Supplementary Fig. 3A, B). Collectively, these results demonstrate that USP10 is a bona fide interacting protein for KLF4.

\section{Loss of USP10 promotes lung tumorigenesis via the downregulation of KLF4}

Previous study has demonstrated that KLF4 is an important tumor suppressor in lung cancer [37], we therefore explored whether USP10 suppressed lung tumor formation via regulating KLF4. To this end, we overexpressed KLF4 in H1299 and A549 cells with or without USP10 KD. Silencing USP10 expression could markedly increase the proliferation and migration in lung cancer cells, and restoration of KLF4 expression completely reversed the phenotypes caused by USP10 KD (Fig. 4a, b and Supplementary Fig. 4A-D). Consistently, Xenograft assays showed that the overexpression of KLF4 could reverse the effects of USP10 depletion and inhibit the tumor development and metastasis (Fig. 4c-g and Supplementary Fig. 4E).

To interrogate the biological role of USP10 in lung tumorigenesis in vivo, the $\mathrm{Kras}^{\mathrm{G}}{ }^{2 \mathrm{D}}$-driven lung $\mathrm{ADC}$ model was established (Supplementary Fig. 4F). Adenoassociated virus expressing USP10-shRNA was delivered into mouse lung to knock down endogenous USP10 expression (USP10 KD) by intratracheal injection. After 2 weeks, adeno-associated virus expressing Crerecombinase-GFP (AAV-Cre-GFP) were delivered into $\mathrm{Kras}^{L S L-G 12 D / W T} /$ Usp10 WT and Kras ${ }^{L L L-G 12 D / W T} /$ sp $10 \mathrm{KD}$ mice by intranasal instillation to induce expression of $\mathrm{Kras}^{\mathrm{G} 12 \mathrm{D}}$ protein. The efficiency of AAV-mediated gene delivery was evaluated using the bioluminescence imaging, and equal introduction of GFP-Cre signals were monitored to exhibit in the lung (Supplementary Fig. 4G). We imaged a separate cohort of $\mathrm{Kras}^{\mathrm{GI2D} / \mathrm{WT}} / \mathrm{Usp10} \mathrm{WT}$ and $\mathrm{Kras}^{\mathrm{GI} D /}$ ${ }^{W T} /$ spp10 KD mice at 30,60, and 120 days post AAV-Cre infection using Micro-CT (Micro-Computed Tomography) imaging. Kras ${ }^{G 12 D / W T} /$ UsplO KD mice showed more detectable nodules than $\mathrm{Kras}^{G 12 D / W T} / \mathrm{Usp} 10 \mathrm{WT}$ mice in the lung by coronal and transverse section imaging $\mu \mathrm{CT}$ scan (Fig. 4h). Subsequently, we performed tumor analysis in mice at 120 days post AAV-Cre-induction. $\mathrm{Kras}^{\mathrm{Gl2D} / \mathrm{WT}}$ / Usp10 KD mice exhibited a dramatical increase in tumor burden and tumor area compared with $\mathrm{Kras}^{\mathrm{GI2D} / \mathrm{WT}} / \mathrm{Usp} 10$ 

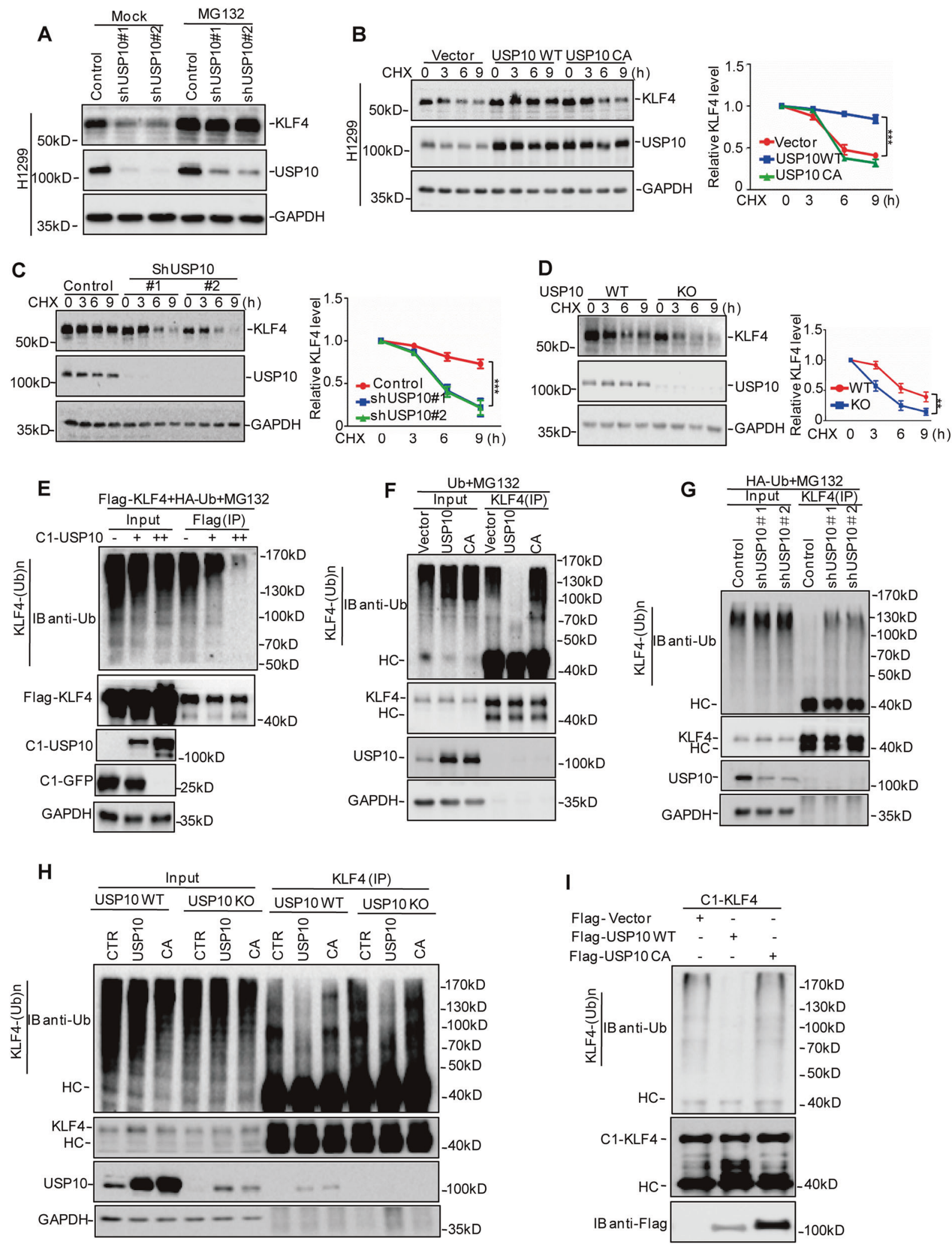

WT mice (Fig. 4i-k). Tumor sections from $\operatorname{Kras}^{G 12 D / W T} /$ Usp10 KD mice displayed increased cell proliferation, amplified angiogenesis and decreased cell apoptosis signals than those of tumor tissues from $\mathrm{Kras}^{G 12 D / W T} / \mathrm{Usp} 10 \mathrm{WT}$ mice, as indicated with the $\mathrm{Ki}-67, \mathrm{CD} 31$, and CleavedCaspase 3 staining (Fig. 41). Importantly, loss of USP10 
Fig. 2 USP10 maintains KLF4 stability and de-polyubiquitylates KLF4. a H1299 cells with or without USP10 knockdown were treated with MG132 for $8 \mathrm{~h}$. Cell lysates were subject to analyze with the indicated antibodies. H1299 cells with or without USP10 WT or CA mutant overexpression (b) or USP10 knockdown (c) or USP10 knockout (d) were treated with CHX $(10 \mathrm{mg} / \mathrm{ml})$ for the indicated times. The half-life of KLF4 was measured. e 293T cells were transfected with the indicated constructs were treated with MG132 for $8 \mathrm{~h}$ before collection. The whole-cell lysates were subjected to immunoprecipitation with Flag antibody and western blot with anti-Ub antibody to detect ubiquitylated KLF4. H1299 cells with or without USP10 WT or CA overexpression (f) or USP10 knockdown (g) were transfected with the indicated constructs. After $24 \mathrm{~h}$, the cells were treated with MG132 for $8 \mathrm{~h}$ before collection. The whole-cell lysates were subjected to immunoprecipitation with KLF4 antibody and western blot with anti-Ub antibody to detect ubiquitylated KLF4. h The indicated constructs were transfected into H1299 cells with or without USP10 knockout and then the cells were treated with MG132 for $8 \mathrm{~h}$ before collection. The whole-cell lysates were subjected to immunoprecipitation with KLF4 antibody and western blot with anti$\mathrm{Ub}$ antibody to detect ubiquitylated KLF4. $\mathbf{i}$ In vitro deubiquitination assay was performed. Ubiquitinated GFP-KLF4 protein was treated with USP10 WT or USP10 CA. Reaction mixes were analyzed by western blotting. For all panels, data are representative results of three independent experiments. b, c, d Quantification of KLF4 levels relative to GAPDH was shown. Results were shown as mean \pm s.d; twoway ANOVA test, $* * p<0.01, * * * p<0.001$

leads to a markedly shortened survival and poor prognosis of $\mathrm{Kras}^{G 12 D / W T} / \mathrm{Usp} 10 \mathrm{KD}$ mice (Fig. 4m). In addition, we detected KLF4 expression in the lung tissue and found that the depletion of USP10 in $\mathrm{Kras}^{G 12 D / W T}$ mice significantly decreased KLF4 protein levels and increased KLF4 ubiquitination (Fig. 4n, o and Supplementary Fig. 4H). Taken together, these results demonstrate that USP10 suppressed tumor growth and metastasis by regulating KLF4 in vivo and in vitro.

\section{KLF4 transcriptionally upregulates TIMP3 expression}

To dissect the possible molecular mechanisms how the effect of USP10 on KLF4 expression suppresses lung cancer initiation and progression, we used RNA-seq to identify the downstream genes of KLF4, which were affected by USP10. After the KO of both genes through the CRISPR/Cas9 system, we detected 629 (183 upregulated and 446 downregulated) and 650 (219 upregulated and 431 downregulated) differentially expressed mRNAs for KLF4 KO and USP10 KO, respectively, under the screening criteria of the absolute $\log 2$-fold change $>1$ and $q$ value $<0.05$ (Supplementary Fig. 5A, B). Not surprisingly, a number of the differentially expressed genes (58 upregulated and 243 downregulated) exhibited overlapping expression in KLF4 KO and USP10 KO conditions (Fig. 5a and Supplementary Fig. 5C). Moreover, we found that some known tumor suppressor genes were concomitantly downregulated after KLF4 KO and USP10 KO (Fig. 5b).

Intriguingly, TIMP3 was revealed to be significantly enriched in many critical signatures representing cancerassociated biological states or processes by computing overlaps between the 243 downregulated genes and gene sets in MSigDB (Supplementary Fig. 5D-F). In particular, among those downregulated genes that can be classed into Module5 (lung cancer gene set), TIMP3 was a top-ranked tumor suppressor gene (Fig. 5c). Subsequently, TIMP3 downregulation was further validated using the q-RT-PCR and western blotting assays in KLF4 KO and USP10 KO lung cancer cells, respectively (Fig. 5d, e). Similar results were obtained when endogenous KLF4 and USP10 were knocked down by two independent shRNAs (Fig. 5f, $\mathrm{g}$ and supplementary Fig. 6A, B). The decrease of TIMP3 by USP10 depletion was reversed by KLF4 overexpression. On the contrary, the increase of TIMP3 by USP10 overexpression was abolished when KLF4 was knocked out (Fig. 5h, i). In addition, the loss of USP10 also suppressed TIMP3 expression in lung tissues and MEF cells from Usp10 KO mice (Fig. 5j and Supplementary Fig. 6C-E).

To identify the KLF4-binding regions on the TIMP3 promoter, the two upstream sequences of TIMP3 were cloned into pGL3-based luciferase reporter plasmids (named P1 and P2), which then were transfected into H1299 with or without KLF4 KO. Compared with the control cells, the luciferase activities of P2 not P1 were decreased in KLF4 KO cells (Fig. 5k, 1). On the contrary, KLF4 overexpression led to an increase of luciferase activity of P2 (Fig. 5m). Thus, these results indicated that the region -500 to $0 \mathrm{bp}(\mathrm{P} 2)$ was a key region for the promotion of TIMP3 transcription by KLF4.

Previous reports have indicated that KLF4 is a zinc finger-type transcription factor that usually binds to the GC rich element of the promoters [38]. To identify the KLF4binding sites, we inspected the sequence of $\mathrm{P} 2$ and found a putative KLF4-binding site. To verify that the potential KLF4-binding site was indeed responsive to KLF4, two pGL3-based luciferase reporter plasmids named BS WT and BS Mut were constructed (Fig. 5n). Then, these plasmids were individually transfected into H1299 cells with or without KLF4 overexpression. As shown in Fig. 5o, the luciferase activity of BS but not BSM was significantly increased in KLF4 overexpressed cells indicating that the binding site was a positive KLF4-binding site in the TIMP3 promoter.

In addition, subsequent chromatin immunoprecipitation (ChIP) assay showed that the chromatin fragments corresponding to the region (P2) were specifically present in the anti-KLF4 immunoprecipitates from H1299 cells. The binding of KLF4 to the TIMP3 promoter was decreased when KLF4 was knocked out, whereas the binding was 


\section{A}

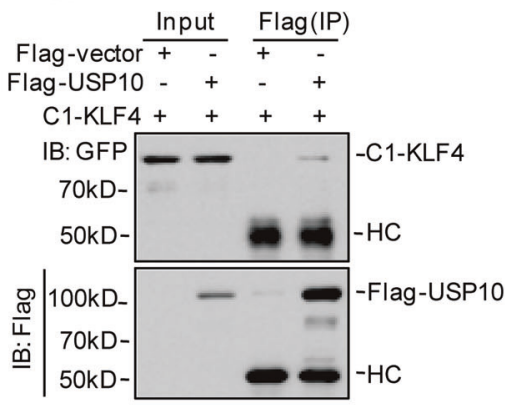

B

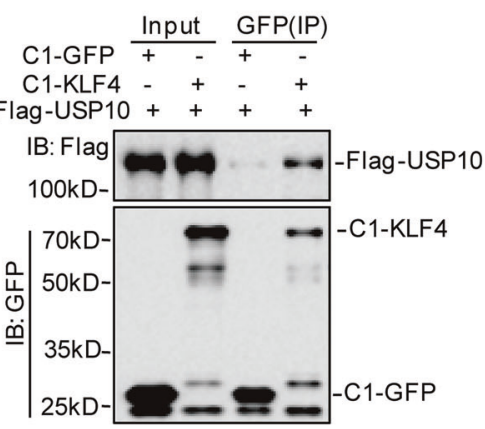

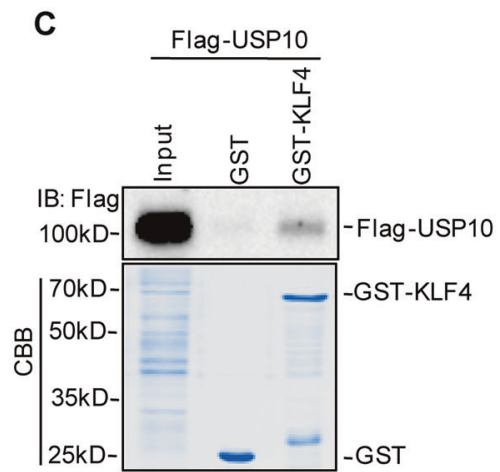

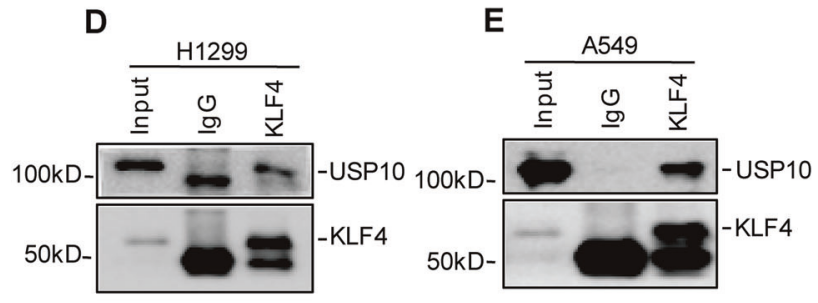

H

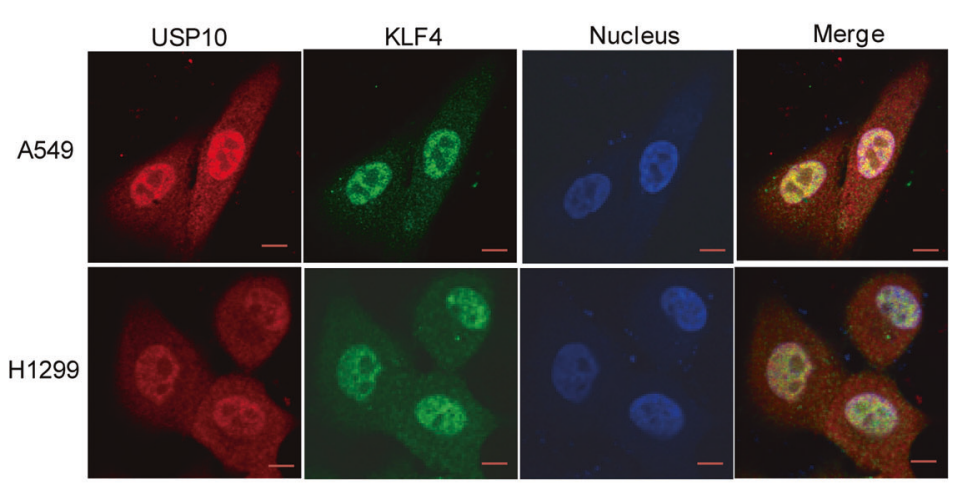

I
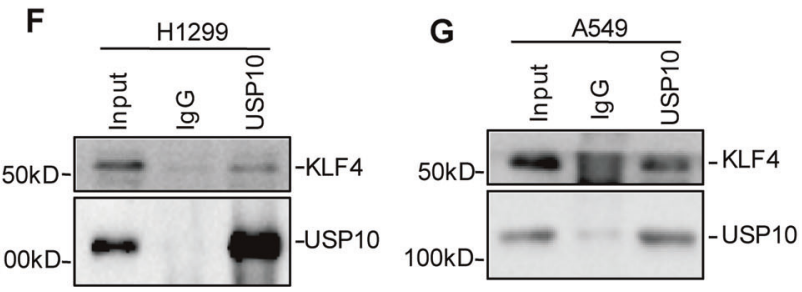

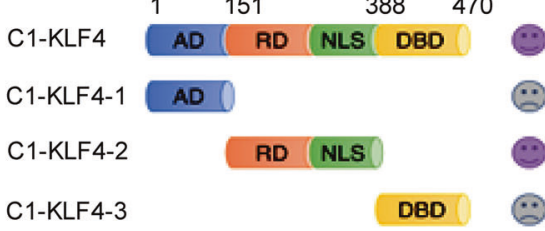

C1-KLF4-3

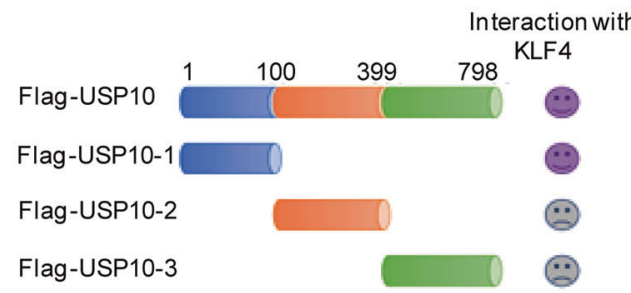

J

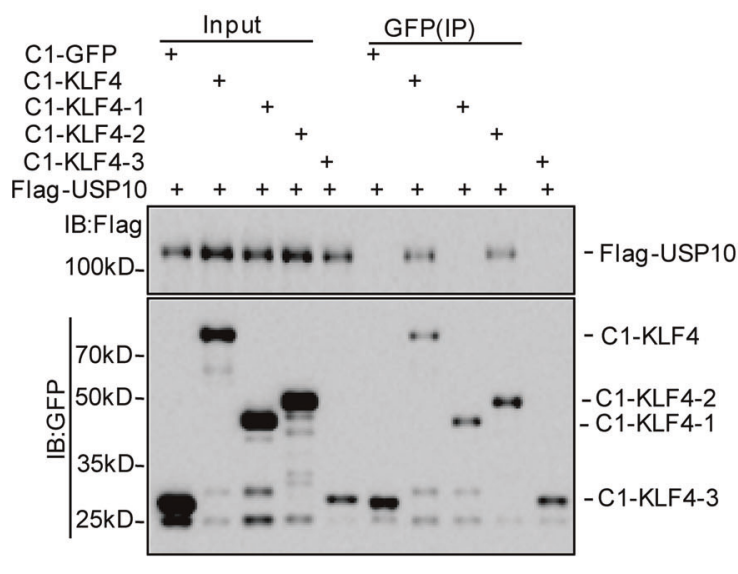

K

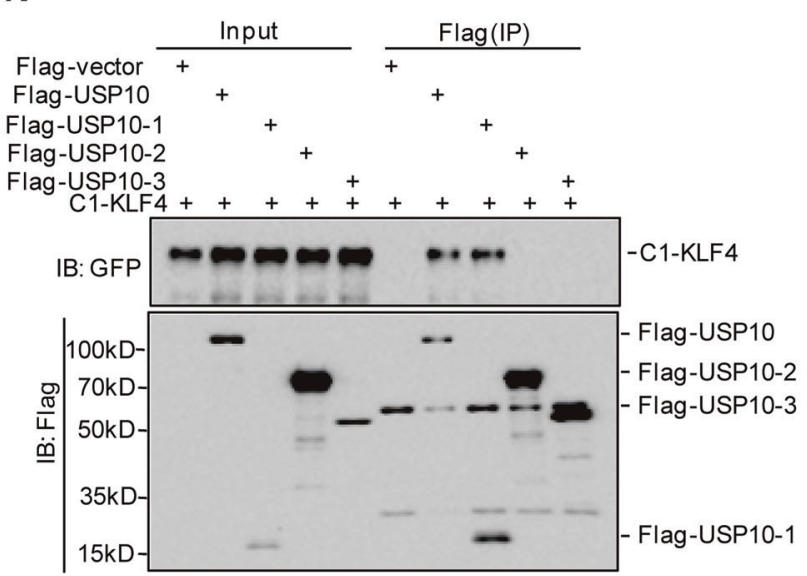


Fig. 3 USP10 interacts with KLF4. a, b Flag-USP10 and C1-KLF4 were co-transfected into 293T cells. The cell lysates were subjected to immunoprecipitation with anti-Flag or anti-GFP antibodies. c Purified Flag-USP10 from 293T cells was incubated with purified recombinant GST-KLF4 or GST for $12 \mathrm{~h}$ at $4{ }^{\circ} \mathrm{C}$. Flag-USP10 retained on Sepharose was blotted with the Flag antibody. d-g H1299 and A549 cell lysates were subject to immunoprecipitation with control IgG, anti-USP10, or anti-KLF4 antibodies. The immunoprecipitates were then detected using the indicated antibodies. h A549 and H1299 cells were fixed and stained with USP10 antibody (Red) and KLF4 antibody (Green). Nuclei were stained with DAPI (blue). Scale bar: $20 \mu \mathrm{m}$. i-k Schematic illustration of KLF4 and USP10 structure. The indicated constructs were transfected into $293 \mathrm{~T}$ cells. After $24 \mathrm{~h}$, the cells were subject to immunoprecipitation with anti-Flag or anti-GFP antibodies. For all panels, data are representative results of three independent experiments

increased when KLF4 was overexpressed (Fig. 5p and supplementary Fig. 6F). Taken together, our data indicate that TIMP3 is a downstream gene of USP10-KLF4 pathway.

\section{USP10 and KLF4 inhibit lung cancer cell growth and metastasis via regulating TIMP3 expression}

To assess whether USP10 and KLF4 inhibits tumorigenesis through upregulating TIMP3 expression, we overexpressed TIMP3 in KLF4 or USP10 depleting-H1299 and A549 cells and observed that the ectopic expression of TIMP3 attenuated KLF4 or USP10 depletion-induced cell growth and migration (Fig. 6a-f and supplementary Fig. 7A-F). Consistently, xenograft assays showed that the overexpression of TIMP3 could rescue the effects of KLF4 or USP10 loss and inhibit the tumor development (Fig. 6g-j). Collectively, these results demonstrate that USP10 and KLF4 inhibit tumor growth and metastasis by upregulating TIMP3.

\section{Reduced USP10 positively correlates with the downregulation of KLF4 and TIMP3 and predicts a poor prognosis of lung cancer patients}

To evaluate the clinical importance of the USP10-KLF4TIMP3 axis and determine their correlation in lung cancer, we performed immunohistochemical (IHC) staining to examine the expression of these proteins in serial sections of tissue microarrays (TMAs) containing lung ADC tissues $(n=92)$ and adjacent tissues $(n=87)$. The IHC results showed that compared with the adjacent or matched adjacent tissues, the expression levels of USP10, KLF4, and TIMP3 are significantly downregulated in lung ADC tissues (Fig. 7a-g). In particular, patients bearing lung tumor with relatively low levels of USP10, KLF4, or TIMP3 showed shorter overall survival than those with high levels of these proteins (Fig. 7h, i). Furthermore, our data also indicated that these tumor samples exhibited the positive correlation between the expression levels of USP10, KLF4, and TIMP3 proteins (Fig. $7 \mathrm{k}-\mathrm{n}$ ).

The above findings are further reinforced by analyzing RNA-seq data of two cohorts of lung cancer patients obtained from the TCGA and GEO datasets. We verified that the mRNA levels of KLF4 and TIMP3 are lower in lung tumor tissues than in normal tissues and KLF4 is also positively correlated with TIMP3 expression level (Supplementary Fig. 8A-F). Taken together, these results validate the mechanistic link among USP10, KLF4, and TIMP3 in lung cancer.

\section{Discussion}

KLF4 is a labile protein with a high turnover rate in a wide variety of human cancers [30]. However, mechanisms regulating KLF4 protein stability and activity are largely unknown in lung cancer. In this study, we identify USP10 as a novel DUB that can stabilize KLF4 and block its degradation in lung cancer. Loss of USP10 in mice downregulates KLF4 expression and accelerates lung ADC progression. Importantly, reduction of USP10 expression is positive correlation with decreased KLF4 in lung ADC tissues and predicts poor survival of lung cancer patient.

USP10 is a member of the ubiquitin-specific protease family that can remove ubiquitins from its substrates. Yuan et al. found that phosphorylation of USP10 on Thr-42 and Ser-337 by ATM leads to USP10 translocation into the nucleus to stabilize p53 [39]. Besides p53, some other substrates of USP10 were also reported, including sorting nexin 3 , the cystic fibrosis transmembrane conductance regulator, H2A.Z, MutS Homologs2, topoisomerase II $\alpha$, $\mathrm{AMPK} \alpha, \mathrm{p} 14 \mathrm{ARF}$, and the NOTCH1 intracellular domain [40-47]. In consideration of these substrates, USP10 was indicated to play an important role in progression of numerous aggressive cancers.

Here, we present several lines of evidence to support the concept that USP10 is a true deubiquitinase for KLF4 and exhibits a tumor suppressive role in lung cancer. Firstly, we identified that USP10 directly interacted with KLF4. Overexpression of USP10 not USP4, USP6, and USP7 remarkably increases KLF4 protein level and decreases its ubiquitination, whereas the depletion of USP10 promotes KLF4 degradation in vitro and in vivo. Although USP4, USP6, and USP7 also interacted with KLF4, they may affect other biological processes of KLF4 such as subcellular localization or transcriptional activity, which will be investigated in future. The KLF4 protein is frequently dysregulated in many tumors. Previous studies indicated that the E3 ligases pVHL and FBXO32 promote KLF4 ubiquitination and degradation in breast cancer [33, 35]. $\beta$-TrCP ubiquitinates KLF4 in mouse embryonic stem cell 
A

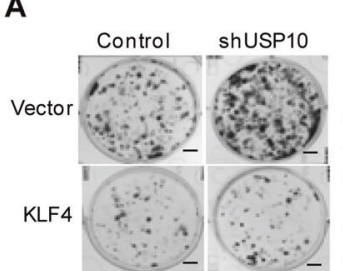

C Control

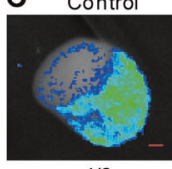

$4 / 6$

E

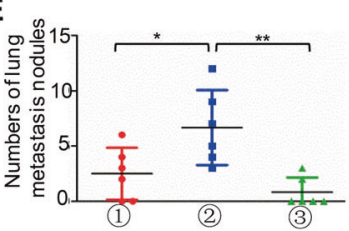

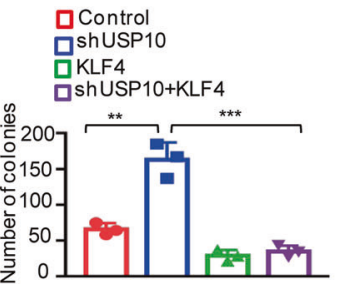

B
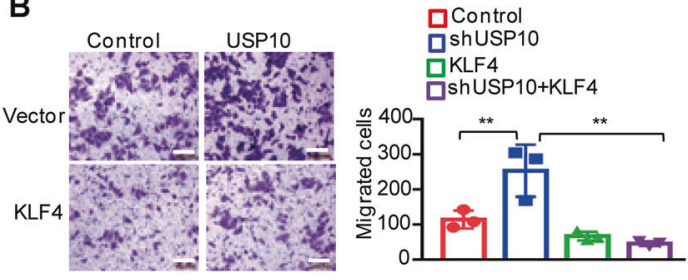

$\mathrm{H}_{\mathrm{A}}$

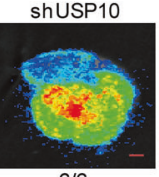

$6 / 6$

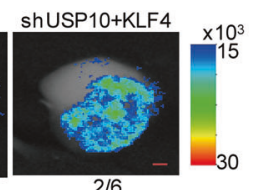

$2 / 6$

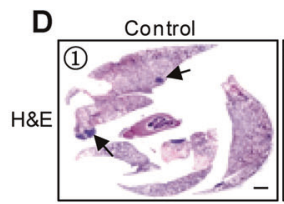

shUSP10
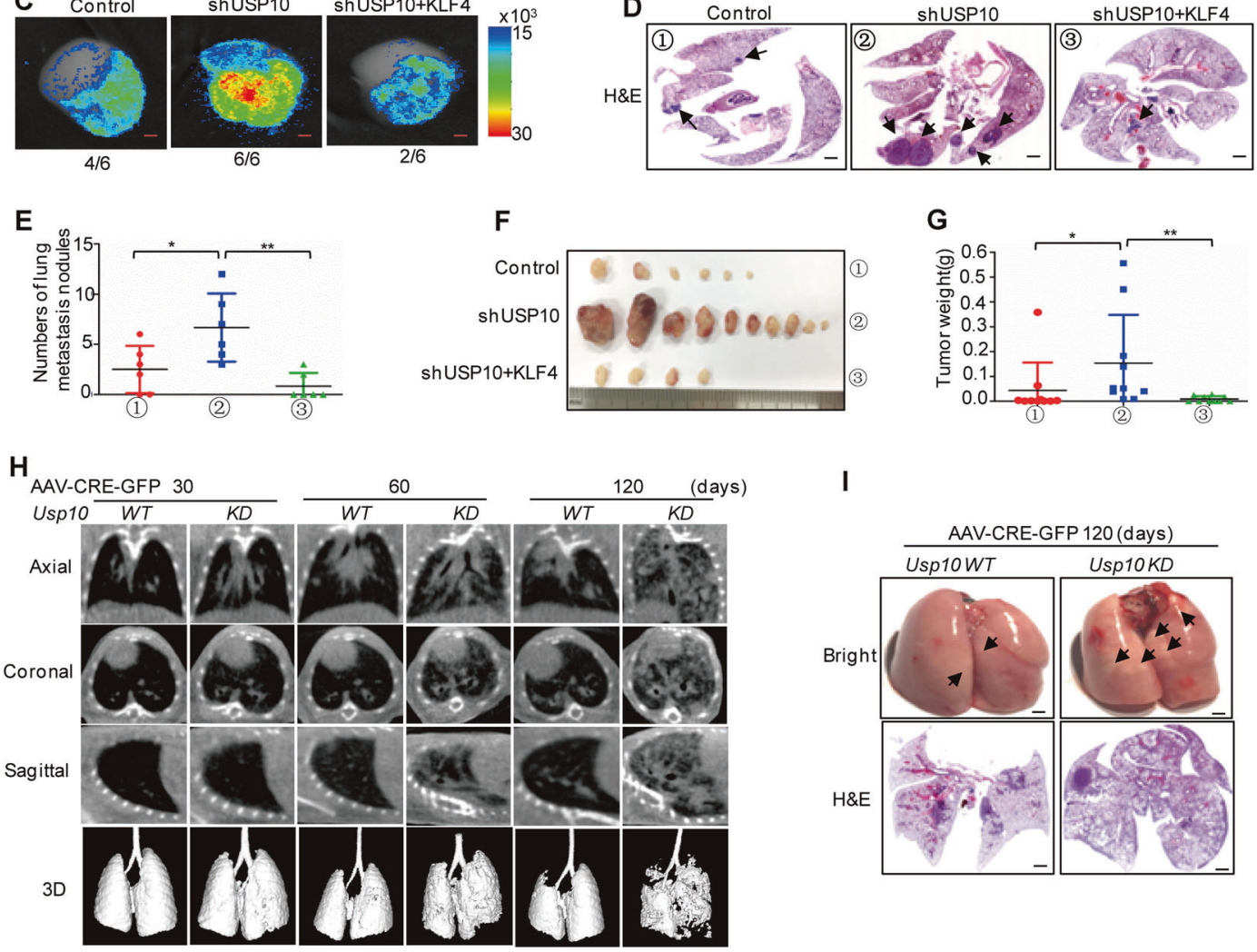

I
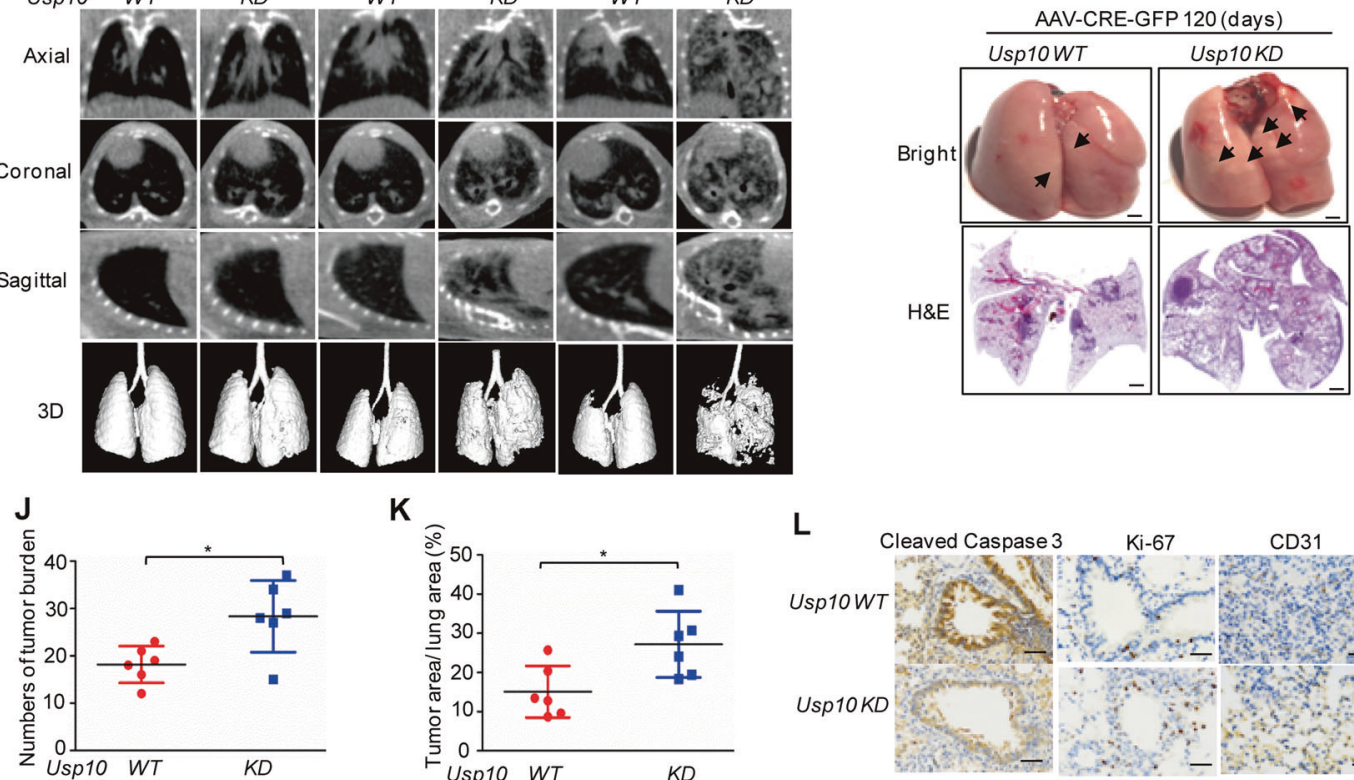

K
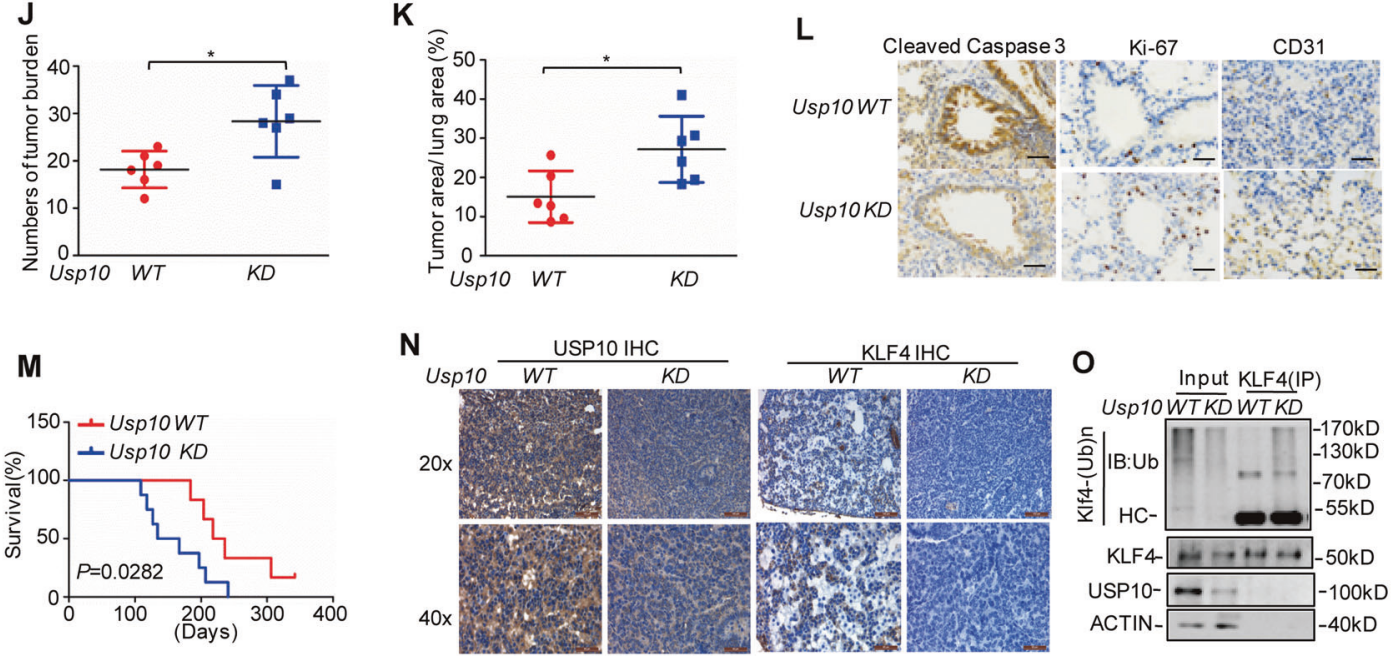

and inhibits stem cell renewal [32]. Mule controls T-cell proliferation by mediating KLF4 ubiquitination and degradation [34]. However, our finding indicated that the reported E3 ligases have no effect on KLF4 expression in lung cancer (Supplementary Fig. 9A-D). It is well known that KLF4 plays a role in the function of tumor suppressor or carcinogenesis in a tissue dependent manner [23]. Like KLF4, its E3 ligases may be altered relying on tumor types. 
Fig. 4 USP10 suppresses tumorigenesis via regulating KLF4 expression. a, b USP10 was knocked down in KLF4-overexpressing H1299 cells. Cell growth and migration were examined by colony formation (a) and transwell assay (b). Scale bars: $1 \mathrm{~cm} \mathrm{(a);} 100 \mu \mathrm{m}$ (b). c-e USP10 was knocked down in GFP-labeled H1299 cells with or without KLF4 overexpression. The cells were intravenously injected into nude mice $(n$ $=6$ in each group). The lung was subjected to bioluminescent imaging (c). Representative images of the lung and HE pictures are shown in d, the metastasis nodule in each group were calculated (e). Scale bars: 2 $\mathrm{mm}(\mathbf{c}) ; 6 \mathrm{~mm}(\mathbf{d}) . \mathbf{f}, \mathbf{g}$ The indicated cells were subcutaneously injected $\left(2.5 \times 10^{6}\right.$ cells per mouse) into nude mice $(n=10)$. Tumor formation was analyzed. h Micro-CT images in indicated planes from the mice with or without USP10 knockdown in lung as indicated days post infection (AAV-shRNA Control or shRNA USP10 together with $1 \times$ $10^{10} \mathrm{pfu}$ AAV-GFP-Cre). Three-dimensional rendering of micro-CT data with lungs in gray, lost part represented tumor. $\mathbf{i}-\mathbf{k}$ Representative images of lung lesions and $\mathrm{H} \& \mathrm{E}$ staining from the indicated experimental groups $(n=6)$. The tumor burden and tumor area were calculated. Scale bars: $2 \mathrm{~mm}($ up); $6 \mathrm{~mm}(\mathrm{dn})$. I Immunohistochemistry analysis protein levels of Cleaved Caspase 3, Ki-67 and CD31 expression in lung tumors of Kras ${ }^{G 12 D / W T} / U_{s p 10}$ WT and $\mathrm{Kras}^{\mathrm{GI} D / W T}$ / Usp10 KD mice, respectively. Scale bars: $50 \mu \mathrm{m}$. m Kaplan-Meier plot showing overall survival of mice with the indicated genotypes infected with AAV-Cre $\left(\mathrm{Kras}^{G I 2 D / W T} / \mathrm{Usp} 10 \mathrm{WT}, \mathrm{n}=6 ; \mathrm{Kras}^{G 12 D / W T} / U \mathrm{sp} 10\right.$ $K D, n=8)$, log-rank test. $\mathbf{n}$ Immunohistochemistry analysis protein levels of USP10 and KLF4 expression in lung tumors. Scale bars: 50 $\mu \mathrm{m}$. o The ubiquitylation of KLF4 was detected in lung tissues. a, b Results are representative of three independent experiments, the data are shown as mean \pm s.d. Student's $t$-test. e, $\mathbf{g}, \mathbf{j}, \mathbf{k}$ Metastasis nodule, tumor weight, tumor burden, and tumor area were analyzed, two-way ANOVA test, ${ }^{*} p<0.05, * * p<0.01, * * * p<0.001$

Thus, the potential E3 ligases of KLF4 in lung cancer still need further investigation.

Secondly, KD of USP10 promoted lung cancer growth and migration by downregulating KLF4 expression. Previous studies indicated that KLF4 is mainly expressed in fibroblasts and airway epithelial cells in the lung tissue, and is the most significantly increased lung gene at birth [48]. Depletion of KLF4 in mice promotes lung tumor formation [37]. Consistently, KO of USP10 in mice downregulates KLF4 expression and accelerates Kras ${ }^{\mathrm{G} 12 \mathrm{D}}$-driven lung ADC initiation and progression. The subsequent RNA-seq analysis indicated that the tumor suppressor TIMP3 is a downstream target gene of KLF4 and deficiency of USP10 or KLF4 downregulates TIMP3 expression in lung cancer. TIMP3, one member of TIMP families, exerts antitumor effect via suppressing the activity of matrix metalloproteinases or promoting the caspase activation [49]. In lung cancer, several genes have been reported to contribute to the downregulation of TIMP3. The Histone-lysine N-methyltransferase EZH2 can silence TMIP3 expression through the elevation of TIMP3 promoter methylation [50]. The miR-221/222 directly targets TIMP3 and blocks its translation [51]. We uncovered a new regulatory mechanism of TIMP3 expression in lung cancer and found that KLF4 could directly bind to the promoter of TIMP3 and facilitate its transcription.
Finally, the protein levels of USP10, KLF4, and TIMP3 are significantly reduced in lung cancer tissues compared with the adjacent tissues, and USP10 expression correlates with the KLF4 protein levels in lung cancer tissues. The patients bearing lung tumors with relatively low levels of USP10, KLF4, or TIMP3 owned poorer overall survival. In addition, our data also suggested that the mRNA levels of KLF4 are downregulated in lung cancer tissues and are positively associated with TIMP3 mRNA expression. Previous study has demonstrated that mutations and promoter methylation are not the major mechanisms that cause the downregulation of KLF4 mRNA in lung cancer [37]. Although we found that USP10 is an important regulator of KLF4 protein, the decrease of KLF4 mRNA in lung cancer is still not completely clear and thus, the precise mechanisms need to be elucidated in future.

Collectively, our study provides a new insight into the regulatory mechanism of KLF4 in lung cancer and uncovers that USP10 is a novel DUB for KLF4 depolyubiquitylation. Functional study reveals that the loss of USP10 expression facilitates lung tumorigenesis in vitro and in vivo. Moreover, this observation further verified TIMP3 as a downstream target gene of KLF4 in lung cancer. In conclusion, our results demonstrate that USP10 is an essential regulator of KLF4 and that the USP10-KLF4TIMP3 signaling axis plays a critical role in lung cancer suppression.

\section{Materials and methods}

\section{Cell culture and reagents}

NCI-H1299 (H1299), A549, HBE, and HEK293T cell lines were obtained from the American Type Culture Collection. HBE and HEK293T cells were cultured in DMEM (GIBCO-Invitrogen) supplemented with $10 \%$ fetal bovine serum (FBS). A549 and H1299 cells were cultured in 1640 (GIBCO-Invitrogen) supplemented with 10\% FBS. USP10 WT and KO MEF cells were isolated from E13.5 embryos of mice and cultured in DMEM supplemented with $10 \%$ FBS and $100 \mu \mathrm{g} / \mathrm{ml}$ streptomycin. Reagents used in this study included MG132 (Calbiochem), Puromycin (Mediatech), CHX (Inalco Spa Mllano Italy), and Polybrene (Sigma).

\section{Plasmids and antibodies}

The human deubiquitinase expression plasmids used in the article were purchased from OriGene. Full-length USP10 WT, full-length USP10 CA, full-length KLF4 were cloned into the pFlag-CMV-2 or pEGFP-C1 vectors as indicated. The USP10 CA expression plasmid was constructed as 
A
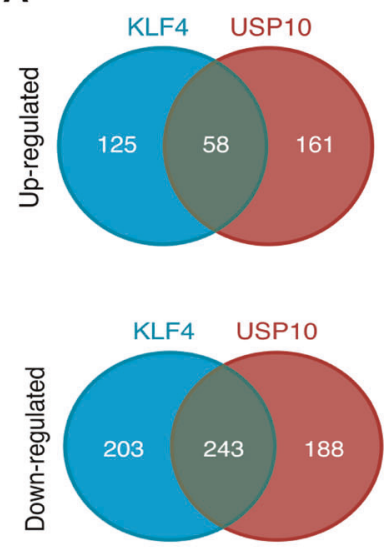

B

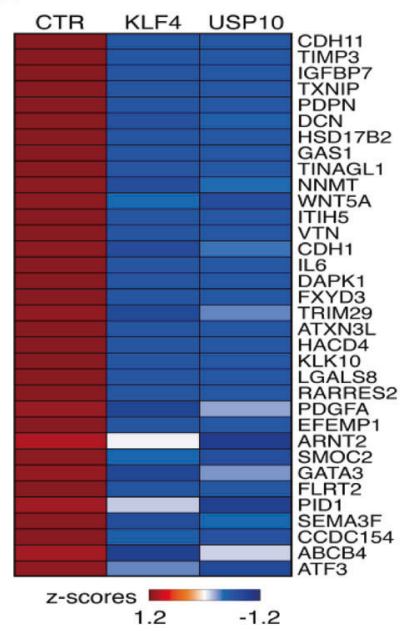

E

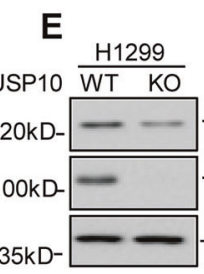

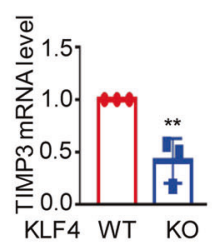

C
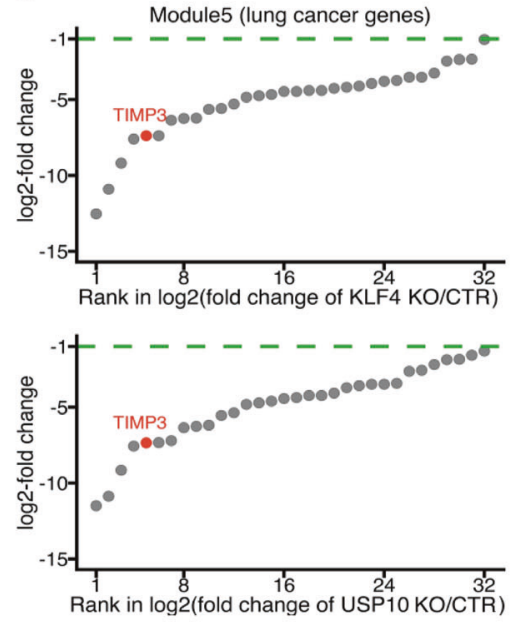

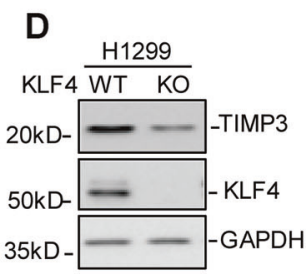

G

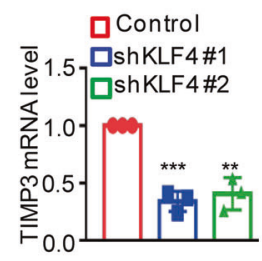

I
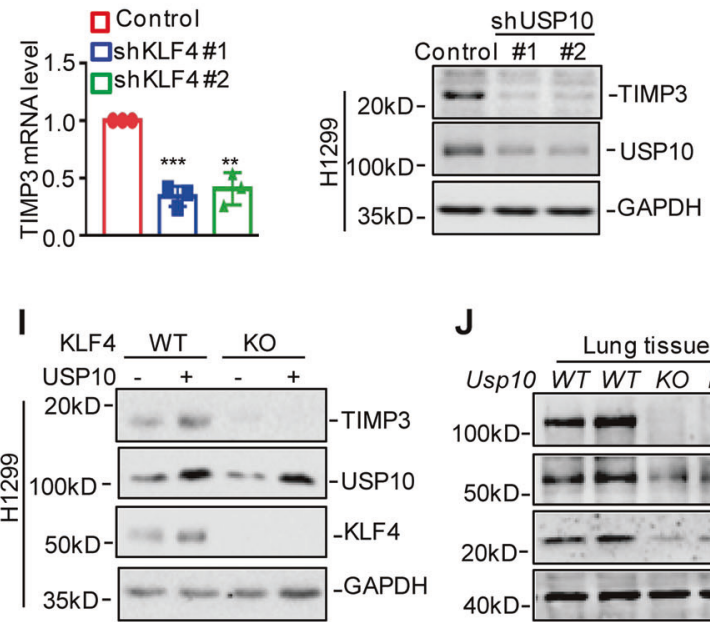

$\mathbf{J}$

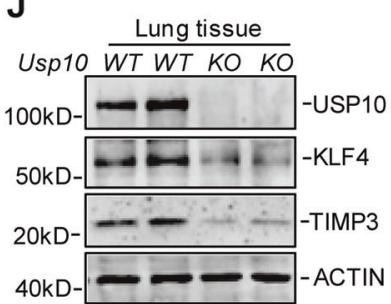

K
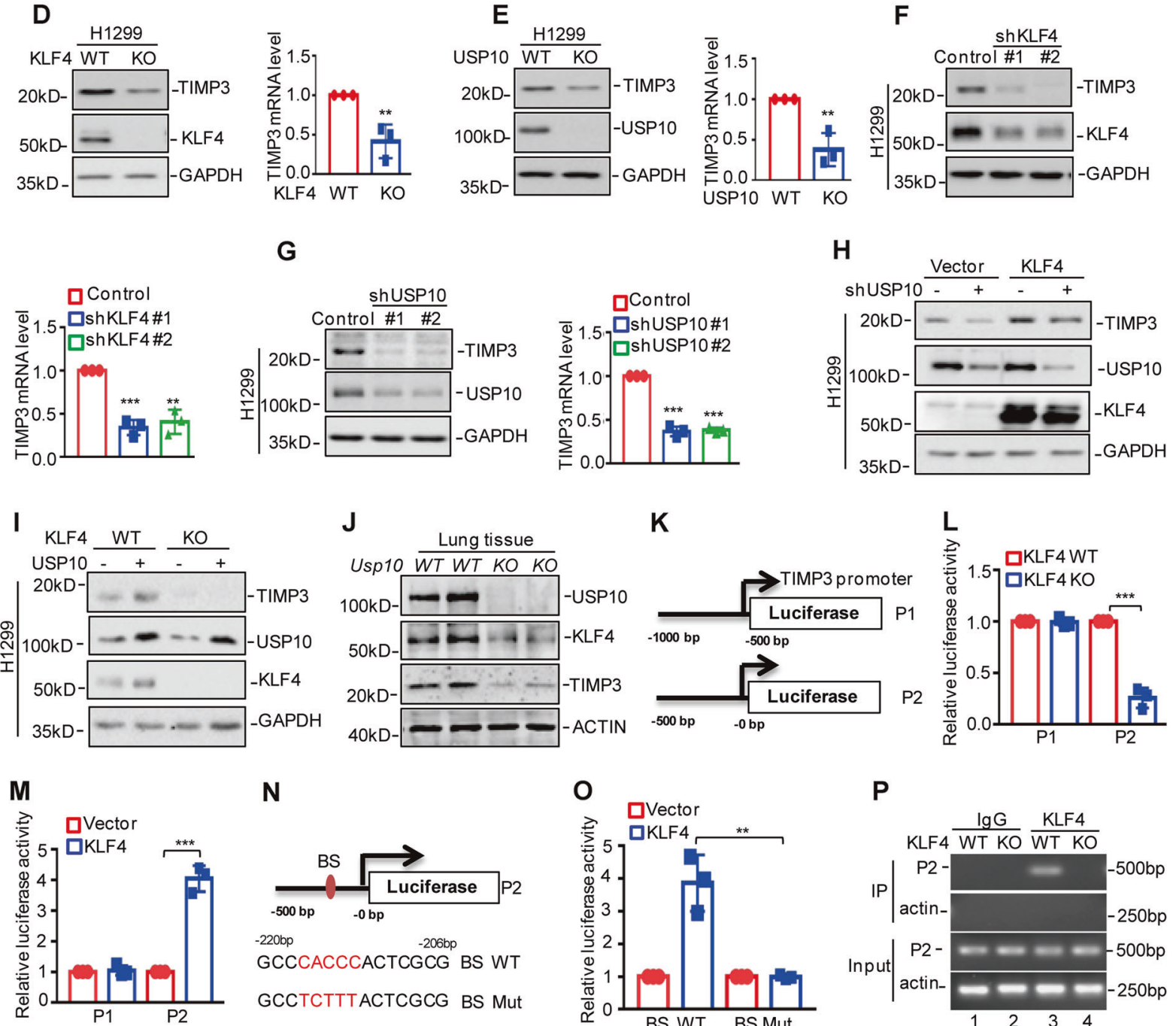

$\mathbf{N}$
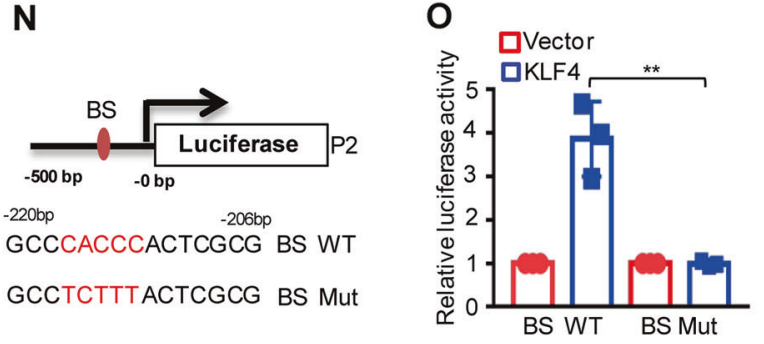

previously described [39]. GST-tagged KLF4 was cloned into the pGEX-4T-2 vector. C1-KLF4-1(aa1-151), KLF4-2 (aa151-388), KLF4-3(aa388-470) were cloned into the pEGFP-C1 vector. Flag-USP10-1(aa1-100), Flag-USP10-2 (aa100-399), and Flag-USP10-3(399-798) were cloned into pFlag-CMV-2 vector. The antibodies used as followed: anti-Actin (1:1,000, sc-1616, Santa Cruz Biotechnology); anti-GAPDH $\quad(1: 1,000, \quad$ SC-25778, Santa Cruz 
Fig. 5 KLF4 upregulates TIMP3 expression. a Overlaps indicating numbers of the differentially expressed mRNAs between KLF4 KO and USP10 KO conditions. b Heatmap showing the expression changes of some known tumor suppressor genes coincidently downregulated after KLF4 KO and USP10 KO. c TIMP3 was the fifth ranked gene among those downregulated genes related to Module5 (lung cancer gene set) in supplementary Fig. 4D. d, e The protein and mRNA levels of TIMP3 were analyzed in H1299 cells with or without KLF4 or USP10 knockout. f, $\mathbf{g}$ The protein and mRNA levels of TIMP3 were analyzed in H1299 cells with or without KLF4 or USP10 knockdown. h USP10 was knocked down in H1299 cells with or without KLF4 overexpression. The expression of TIMP3 was detected by western blotting. i USP10 was overexpressed in H1299 cells with or without KLF4 knockout. The expression of TIMP3 was detected by western blotting. j Immunoblotting analysis the expression levels of TIMP3 in lung tissues from Usp10 WT and $K O$ littlemates. k Schematic illustration of pGL3-based reporter constructs were used in luciferase assays to examine the transcriptional activity of TIMP3. I, $\mathbf{m}$ Parts of the promoter of TIMP3, named P1 and P2 were individually transfected into H1299 cells with or without KLF4 knockout or overexpression. Luciferase activity was measured. n Schematic illustration of KLF4 wild type binding site (BS) and the matching mutant (BSM) that were used in luciferase assays. o The wild type promoter (BS) or the matching mutant (BSM) were individually transfected into H1299 cells with or without KLF4 overexpression. The luciferase activity was measured. p ChIP analysis showed the binding of KLF4 to the promoter of TIMP3 in H1299 cells with or without KLF4 knockout. An isotype-matched IgG was used as a negative control. $\mathbf{d}-\mathbf{j}, \mathbf{l}, \mathbf{m}, \mathbf{o}$ Results are representative of three independent experiments, the data are shown as mean \pm s.d. Student's $t$-test. $* * p<0.01, * * * p<0.001$

Biotechnology); anti-Flag (1:1000; F3165, Sigma); anti-HA (1:1000; sc-1616, Santa Cruz Biotechnology); anti-GFP (1:1000; 66002-1-Ig, Proteintech); Normal rabbit IgG (sc2003, Santa Cruz Biotechnology); anti-KLF4 (1:1000 sc20691 Santa Cruz Biotechnology), anti-KLF4 (1:100, Cell Signaling Technology, \#12173 S), anti-KLF4 (1:500, ab129473, Abcam), Anti-USP10 (1:3000, ab72486, Abcam), Anti-TIMP3 (1:1000, ab39184, Abcam), and antiUb (1:1000, SC-47721, Santa Cruz Biotechnology).

\section{Lentivirus packaging and infection}

To generate the lentiviral shRNA constructs against human KLF4, USP10, $\beta$-TrCP, pVHL, FBXO32, and Mule, the target sequences were cloned into pLKO.1-puro vector or FG12 vector. The shRNA sequences are listed in Table 1.

KLF4 and USP10 KO by CRISPR/Cas9: sgRNA design and cloning was performed according to the Feng Zhang laboratory general cloning protocols. KLF4 and USP10 sgRNAs oligonucleotides were designed based on the target site sequence ( $20 \mathrm{bp}$ ) and were flanked on the $3^{\prime}$ end by a 3 bp NGG PAM sequence. Using the Cas 9 target design tools (http://www.genome-engineering.org), we designed two sgRNAs for each target. The sequences are reported in Table 1. The sgRNAs were cloned into the lentiCRISPRv2 vector (Addgene).

\section{Immunoprecipitation and GST pulldown}

For immunoprecipitation, cells were lysed with 1\%NP40 lysis buffer together with protease-inhibitor cocktail (Biotool). Cell lysates were incubated with the indicated primary antibodies and protein $\mathrm{A} / \mathrm{G}$ agarose beads (Santa Cruz) at $4{ }^{\circ} \mathrm{C}$. The immunocomplexes were then washed with $200 \mu \mathrm{l}$ PBS for twice. Both lysates and immunoprecipitates were examined using the indicated primary antibodies.

GST pulldown assay: GST-KLF4 or GST-tag were purified from the Escherichia coli strain BL21 (Invitrogen) using GST agarose beads. GST or GST-KLF4 bound to GST agarose beads were incubated with Flag-USP10 purified from the HEK293T cells for $6 \mathrm{~h}$ at $4{ }^{\circ} \mathrm{C}$. Then the beads were washed with PBS four times, followed by western blotting.

\section{Protein half-life assay}

For the KLF4 half-life assay, the H1299 and A549 cells with or without USP10 overexpression or depletion were treated with $\mathrm{CHX}$ (Sigma, $10 \mathrm{mg} / \mathrm{ml}$ ) for the indicated durations before collection.

\section{In vivo and in vitro KLF4 deubiquitylation assay}

For KLF4 deubiquitylation in vivo, HA-Ub was transfected into H1299 and A549 cells with or without USP10 overexpression or depletion. The cells then were treated with $20 \mu \mathrm{M}$ of the proteasome inhibitor MG132 (Calbiochem) for $8 \mathrm{~h}$. These cells were lysed with NP40 lysis buffer and incubated with the indicated primary antibodies. For in vitro deubiquitylation experiments, C1KLF4 was transfected into HEK293T cells together with HA-Ub. After $24 \mathrm{~h}, \mathrm{KLF} 4$ was enriched by GFP antibodies. Flag-USP10 and Flag-USP10 CA were expressed in HEK293T cells and purified using Flag-M2 beads. The purified proteins were eluted with $150 \mathrm{ng} / \mathrm{ml}$ FLAG peptide. The proteins were then incubated with GFP-KLF4 in deubiquitylation buffer (50 mM Tris- $\mathrm{HCl}, \mathrm{pH} 7.4,1 \mathrm{mM}$ $\mathrm{MgCl}_{2}$, and $1 \mathrm{mM} \mathrm{DTT}$ ) at $37^{\circ} \mathrm{C}$ for $2 \mathrm{~h}$. Then the beads (GFP-KLF4) were washed with PBS four times, followed by western blot analysis.

\section{Colony formation and cell migration assays}

For colony formation assay, H1299 and A549 cells overexpressing with the indicated genes were diluted the singlecell suspension and 500 or 1000 cells were cultured in every well of 6-well plate at $37{ }^{\circ} \mathrm{C}$ with $5 \% \mathrm{CO}_{2}$ incubator for 2 weeks. Then the colonies were stained with $0.04 \%$ crystal violet- $2 \%$ ethanol and counted. 
A

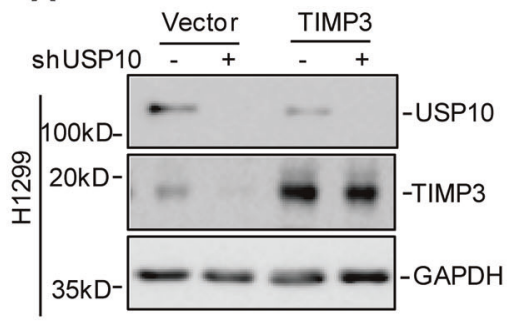

B

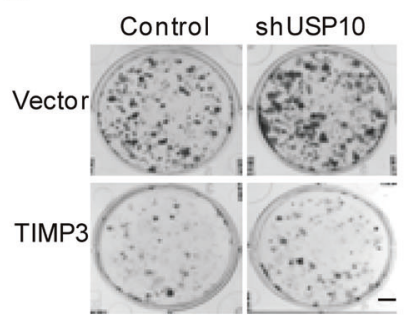

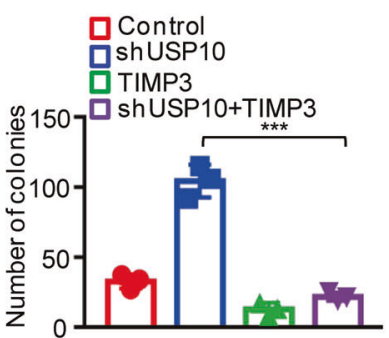

D

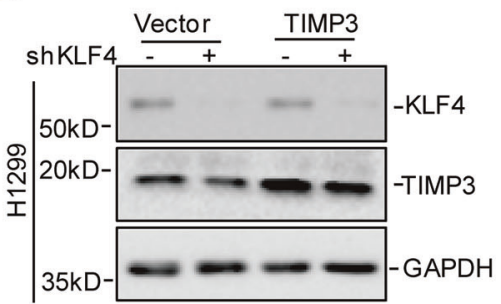

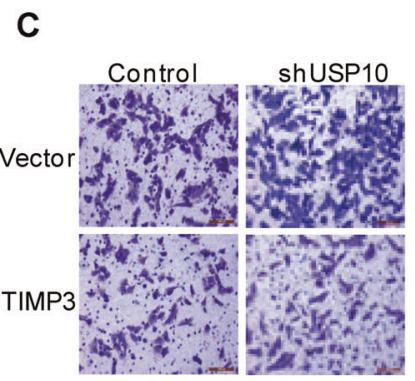

E
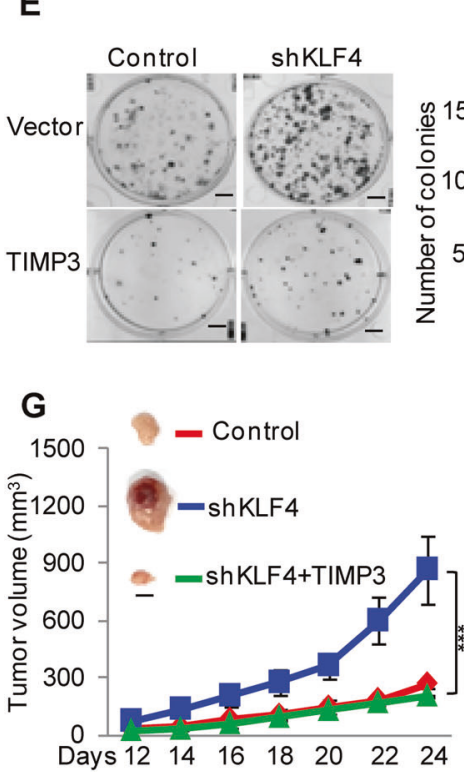

H

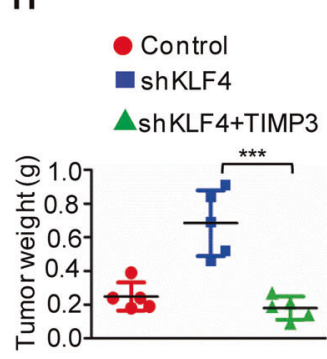

Fig. 6 USP10 inhibits tumorigenesis via regulating KLF4-TIMP3 axis in lung cancer. a-c TIMP3 was overexpressed in H1299 cells with or without USP10 knockdown. The expression levels of USP10 and TIMP3 were analyzed by western blotting (a). Cell growth and migration were examined by colony formation (b) and transwell assays (c). Scale bars: $1 \mathrm{~cm}(\mathbf{b}) ; 100 \mu \mathrm{m}(\mathbf{c})$. d-f TIMP3 was overexpressed in H1299 cells with or without KLF4 knockdown. The expression levels of KLF4 and TIMP3 were analyzed by western blotting (d). Cell

For cell migration assay, the assay was performed in 24well transwell plate with 8 -mm polyethylene terephalate membrane filters (Corning). Cells were allowed to migrate for $24 \mathrm{~h}$ in a humidified chamber. After the incubation period, the filters were removed and fiwer with $4 \%$ formaldehyde for 15 min and cells located in the lower filters were stained with $0.1 \%$ crystal violet for $20 \mathrm{~min}$ and counted.
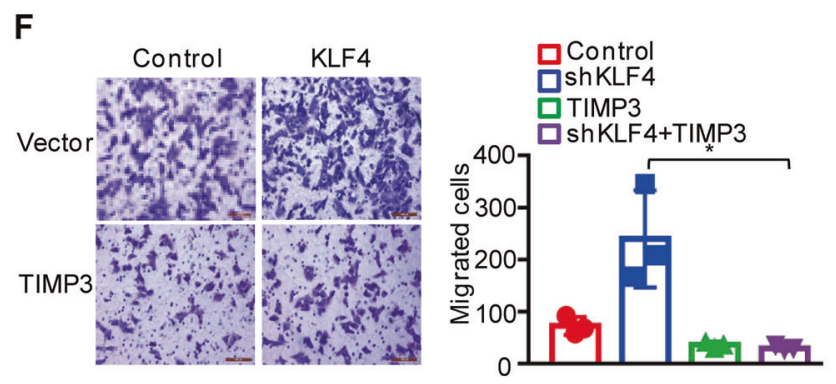

I

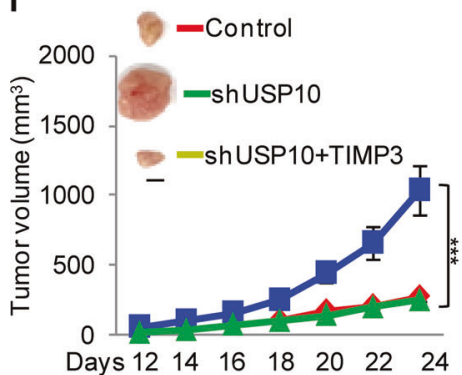

$J$

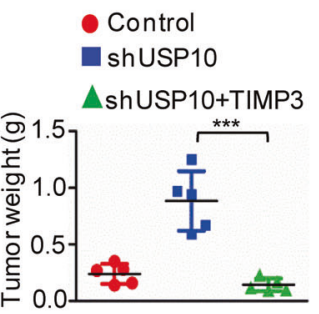

growth and migration were examined by colony formation (e) and transwell assays (f). Scale bars: $1 \mathrm{~cm} \mathrm{(e);} 100 \mu \mathrm{m}(\mathbf{f}) . \mathbf{g}-\mathbf{j}$ The indicated cells were subcutaneously injected $\left(5 \times 10^{6}\right.$ cells per mouse) into nude mice $(n=6)$. Tumor volume and weight were calculated. Scale bars: $0.5 \mathrm{~cm}$. a-f results are representative of three independent experiments, the data are shown as mean \pm s.d. Student's $t$-test. g-i Tumor volume and weight were analyzed, two-way ANOVA test; $* p<0.05, * * * p<0.001$

\section{In vivo tumorigenesis and metastasis assay}

Animal studies were conducted in accordance with the National Institute of Health Guide for the Care and Use of Laboratory Animals with the approval of the Animal Research Committee of Dalian Medical University. Male nude mice (4-6 weeks of age, 18-20 g) were obtained from 


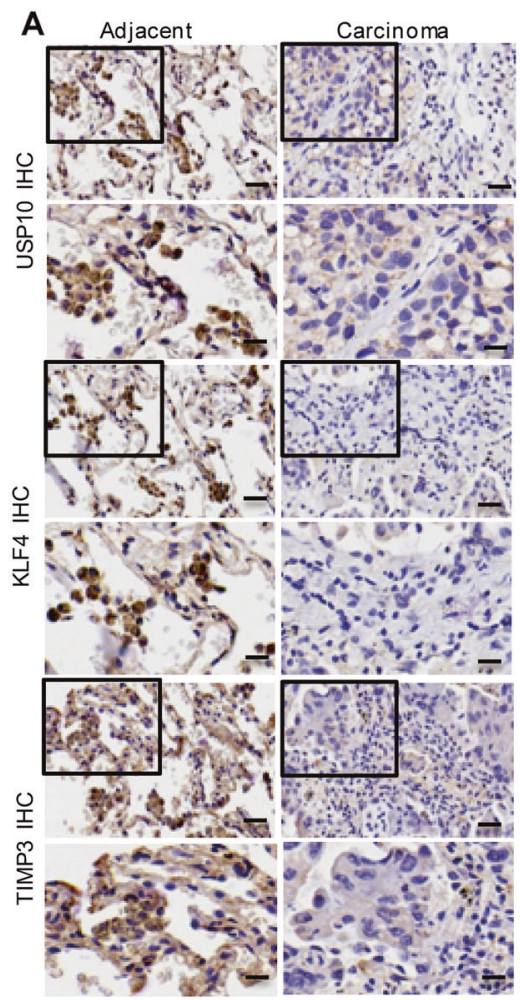

\section{B}

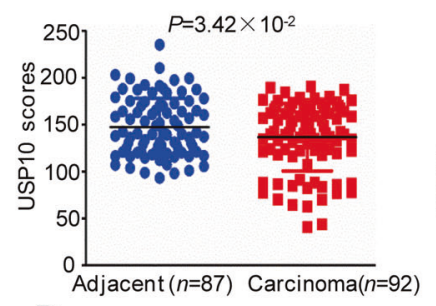

D

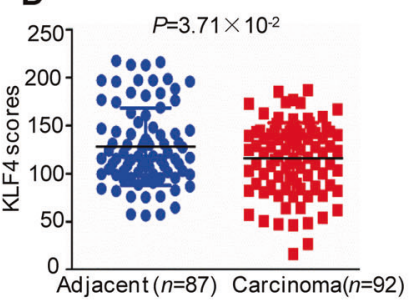

$\mathbf{F}$

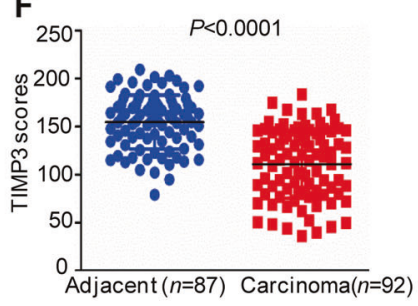

C
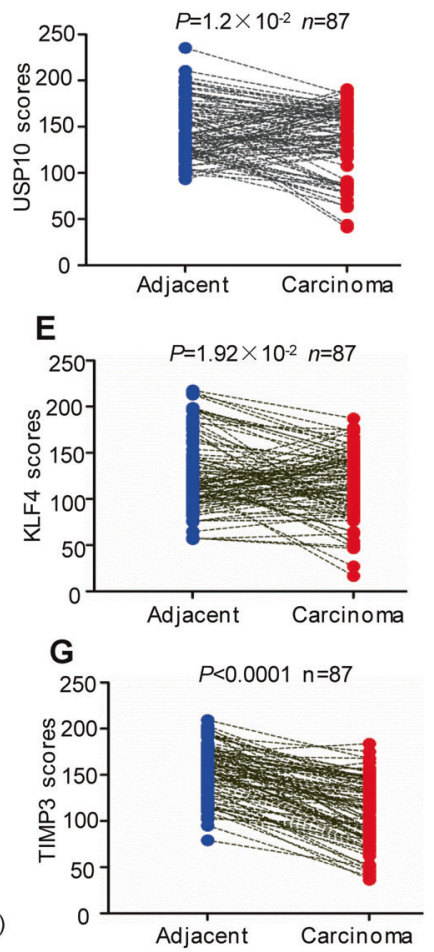
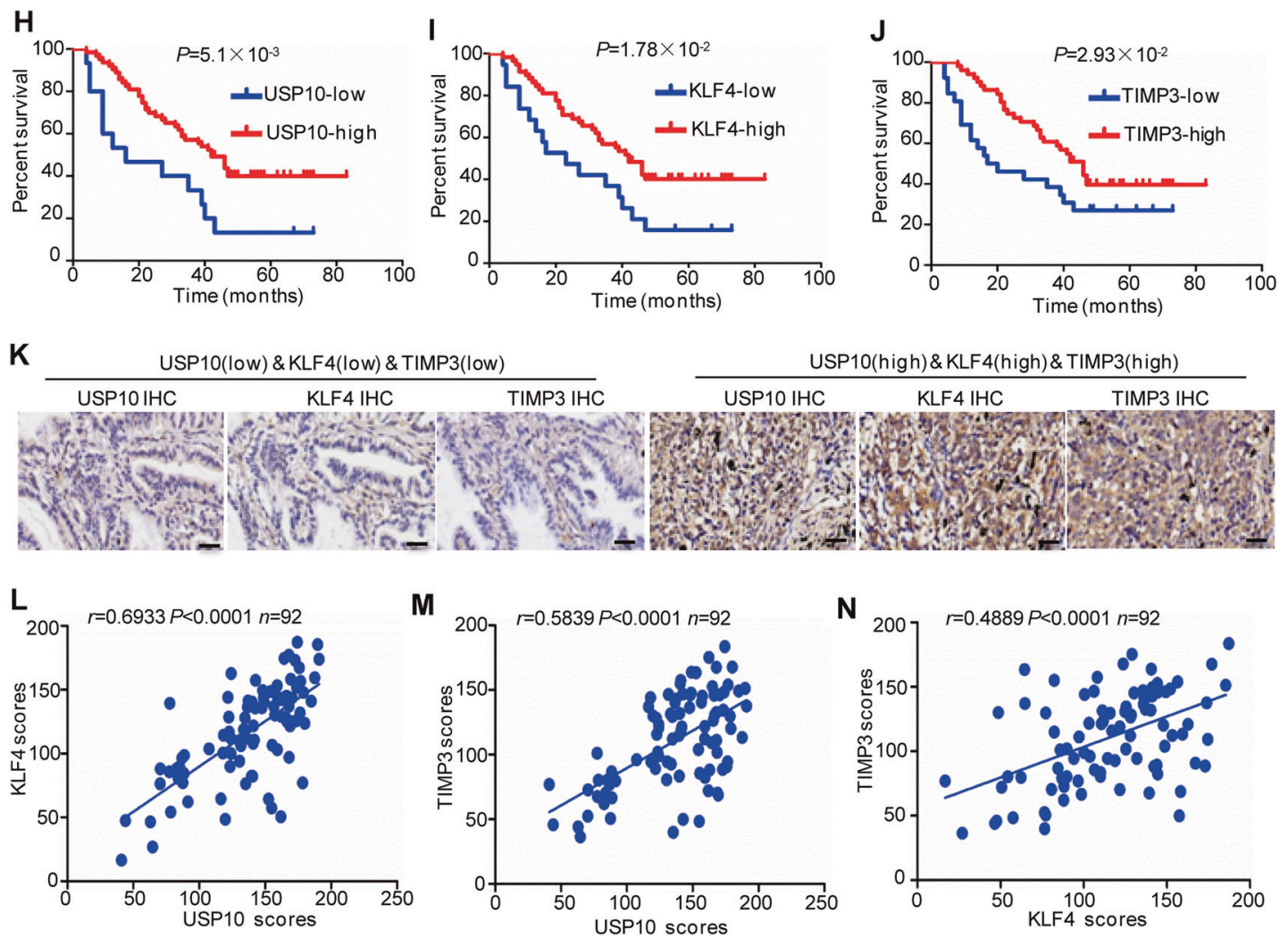

Fig. 7 USP10 is positively correlated with KLF4 and TIMP3 expression in lung adenocarcinoma. a-g Representative images from immunohistochemical staining of USP10, KLF4, and TIMP3 in Lung adenocarcinoma $(n=92)$ and matched adjacent tissue $(n=87)$, respectively. We compared cancer tissues with adjacent tissues or matched adjacent tissues using a Wilcoxon unpaired $t$ test or paired $t$ test $(\mathbf{b}-\mathbf{g})$. Scale bars: $50 \mu \mathrm{m}$. $\mathbf{h}-\mathbf{j}$ Kaplan-Meier plot of overall survival of 92 patients with lung adenocarcinoma. Statistical significance was calculated using log-rank test. $\mathbf{k}-\mathbf{n}$ The correlation of USP10 with KLF4 and TIMP3 protein levels were analyzed. Scale bars: $20 \mu \mathrm{m}$. Data were analyzed using Pearson correlation 
Table 1 Target sequences of shRNAs for indivival genes

shRNA targeting KLF4 (human)
$\begin{array}{ll}\text { no.1 } & \text { 5-TTGGTGAGTCTTGGTTCTAA-3 } \\ \text { no.2 } & \text { 5-CAGAACAAATGTGTTTTTCT-3 }\end{array}$

shRNA targeting USP10 (human)

no.1 5-CCTATGTGGAAACTAAGTATT-3

no.2 5-CCCATGATAGACAGCTTTGTT-3

shRNA targeting USP10 (mouse)

no.1 5-CGCAGAGGAGTATCTAGGTTT-3

no.2 5-CCAAACGGTCAAGGTTATTAA-3

shRNA targeting pVHL (human)

no.1 5-TATCACACTGCCAGTGTATAC-3

no.2 5-TAGGATTGACATTCTACAGTT-3

shRNA targeting FBXO32 (human)

no.1 5-CTTGTTGGTGGCTCGACTCGA-3

no.2 5-AGATTCAGCAACTGGTTAAAG-3

shRNA targeting $\beta$-Trcp (human)

no.1 5-GCGTTGTATTCGATTTGATAA-3

no.2 5-GCTGAACTTGTGTGCAAGGAA-3

shRNA targeting Mule (human)

no.1 5-CGACGAGAACTAGCACAGAAT-3

no.2 5-CCACACTTTCACAGATACTAT-3

gRNA for KLF4 (human)

no.1 5-CACCCGCCGGGCCAGACGCGAACG-3

no.2 5-CACCgTCTTTCTCCACGTTCGCGTC-3

gRNA for USP10(human)

no. 1

5-CACCgGCCTGGGTACTGGCAGTCGA-3

the SPF Laboratory Animal Center of Dalian Medical University (Dalian, China) and were randomly divided into indicated groups. The indicated cells $\left(2.5 \times 10^{6}\right.$ or $\left.5 \times 10^{6}\right)$ were subcutaneously injected into the nude mice or injected through the lateral tail vein into the nude mice. After 12 days, the tumor size was measured every 2 days by Vernier callipers and converted to TV according to the following formula: $\mathrm{TV}\left(\mathrm{mm}^{3}\right)=\left(\mathrm{axb}^{2}\right) / 2$, where $\mathrm{a}$ and $\mathrm{b}$ are the largest and smallest diameters, respectively.

\section{Dual-luciferase and chromatin immunoprecipitation assays}

The promoters of TIMP3 were constructed into the pGL3basic vector and these plasmids were transfected into H1299 cells. The luciferase activity was measured with a Promega Dual-Luciferases Reporter Assay kit (Promega E1980) according to the manufacturer's protocols after transfection. Relative Renilla luciferase activity was normalized to firefly luciferase activity.

ChIP assays were performed by using the Millipore ChIP kit (17-371RF) according to the manufacturer's instructions.
Bound DNA fragments were subjected to RT-PCR using specific primers (Table 2).

\section{Mouse model}

The Usp10 KO mouse model was generated by Model Animal Research Center of Nanjing University. Strategy of Usp10 KO mouse model was illustrated in Supplementary Fig. 1G. Usp10 KO mice were identified by PCR of tail-tip genomic DNA, the primers were shown in Supplementary Table 2. For spontaneous lung tumor experiment, C57 $\operatorname{Kras}^{G 12 D / W T}$ mice were obtained from Shanghai Model Organisms Center, Inc. The mice were identified by PCR of tail-tip genomic DNA. The primers were shown in Table 2.

\section{Virus delivery into the lung}

The adeno-associated virus expressing shRNA Control or shRNA USP10 (two different target sequences were listed in supplementary Table 1) or AAV-Cre-GFP were purchased from Hanbio Co.LTD (Shanghai, China). Delivery of adeno-associated virus was performed accordingly to previously established protocol.

\section{Immunohistochemistry and lung histology}

The lung ADC TMAs purchased from SHANGHAIOUTDOBIOTECHCO.LTD Company, contain enough pairs of cancer tumors together with matched adjacent normal tissue. We performed IHC staining for USP10, KLF4, and TIMP3 on the same paraffin-embedded tissue blocks that were used for clinical diagnosis.

\section{Micro-CT imaging and processing}

Micro-CT imaging was performed using standard imaging protocol with a mCT machine (GE Healthcare). In brief, animals were anesthetized using isoflurane and were set up in the imaging bed with a nosecone providing constant isoflurane. A total of 720 views were acquired for each mouse using a soft-tissue fast-scan setting. Raw image stacks were processed for lung reconstruction using the standard ROI tool (MicroView). Rendering and quantification were performed using render volume tool and measurement tool in MicroView. Tumor burden was calculated as the sum of the sizes in $\mathrm{mm}^{3}$ from all detectable tumors per mouse, with six mice per group.

\section{Fluorescence microscopy}

For detection of subcellular localization by immunofluorescence, after fixation with $4 \%$ paraformaldehyde and permeabilization in $0.2 \%$ Triton X-100 PBS, cells were 
Table 2 Primers for the indicated gene products

\begin{tabular}{lll}
\hline & Forward & Reverse \\
\hline USP10 KO mice & TGGTAAACAGTACGGGGTTTCCTA & CTGCATTCTAGTTGTGGTTTGTCC \\
& TTTCGGAGGACACGCTCTTC & GAACATGCCCGACTCTACCC \\
Kras ${ }^{\text {LSL-G12D/wT mice }}$ & TGTCTTTCCCAGCACAGT & GCAGGTCGAGGGACCTAATA \\
Human KLF4 & CTGCATAGTACGCTATACCCTGT & CTGCATAGTACGCTATACCCTGT \\
Human TIMP3 & ACCTACACAAAGAGTTCCCATC & TGTGTTTACGGTAGTGCCTG \\
Human pVHL & TGATGGCAAGATGTACACGG & GAAGTCACAAAGCAAGGCAG \\
Human FBXO32 & TGCCAATATCACACTGCCAG & GTCTTTCTGCACATTTGGGTG \\
Human $\beta$-Trcp & TCCAAAGAGTCGGCAAGTC & ACAAAGGCAGGTCAGTGAAG \\
Human actin & CTTAAATGGACACAAACGAGGC & CAACGCACCAATTCCTCATG \\
Mouse KLF4 & GTTATACTGTTCCCTGGTGTGG & TACCTGCCTTCTCATCCTTTG \\
Mouse TIMP3 & CACCTTCTACAATGAGCTGCGTGTG & ATAGCACAGCCTGGATAGCAACGTAC \\
Mouse actin & CCAGTATACATTCCGCCACAG & TCTGGGCTTCCTTTGCTAAC \\
P2 fro ChIP & TGAAGGCAAGATGTACACAGG & GAGGTCACAAAACAAGGCAAG \\
\hline
\end{tabular}

incubated with antibodies: anti-USP10 (1:200, ab72486, Abcam), anti-KLF4 (1:100, SC-166190, Santa Cruz) for $8 \mathrm{~h}$ at $4{ }^{\circ} \mathrm{C}$. The nuclei were stained with DAPI (Sigma), and images were visualized with a Zeiss LSM 510 Meta inverted confocal microscope.

\section{Real-time RT-PCR}

Total RNA was isolated using Trizol (Invitrogen). One microgram of total RNA was used to synthesize cDNA using the PrimeScript ${ }^{\mathrm{TM}}$ RT reagent kit (Takara, RR047A) according to the manufacturer's instructions. The primers were listed in the Table 2.

\section{RNA-seq}

RNA extraction, library construction, sequencing, and data analysis were performed by Annoroad Gene Technology, Beijing, China.

\section{TCGA analysis}

Gene expression counts of two TCGA cohorts of lung cancer patients quantified by HTSeq were retrieved from GDC data portal (https://portal.gdc.cancer.gov/). In addition, another dataset of 199 NSCLC patient samples was obtained from the NCBI sequence read archive (GEO accession number: GSE81089) and included in our gene expression analysis.

\section{Study approval}

All animals were housed and handled in accordance with the Animal Care and Use Committee at Dalian Medical
University. All animal studies were conducted in accordance with the Dalian Medical University guidelines for animal care, and all animal procedures were approved by the IACUC of the Dalian Medical University (Dalian, China). All studies were performed in accordance with national animal protection laws (certificate number AEE17031). The characteristics of the patients and their tumors were collected through review of medical records and pathologic reports. Informed consent with approval of the ethics committee of Taizhou Hospital of Zhejiang Province was obtained. All of the methods in this study were in accordance with the approved guidelines, and all of the experimental protocols were approved by the ethics committee of Taizhou Hospital of Zhejiang Province.

\section{Statistical analysis}

The statistical significance of differences between various groups was calculated with the two-tailed paired $t$-test, and error bars represent standard deviation of the mean (s.d.). Statistical analyses, unless otherwise indicated, were performed using GraphPad Prism 5 and 7. Data are shown as mean \pm s.d.

Acknowledgements This work was supported by grants from National Natural Science Foundation of China (Nos. 81874136, 81402260), National Key R\&D Program of China (2017YFA0505602), the Liaoning Provincial Natural Science Foundation of China (No. 2015020309).

\section{Compliance with ethical standards}

Conflict of interest The authors declare that they have no conflict of interest. 
Publisher's note Springer Nature remains neutral with regard to jurisdictional claims in published maps and institutional affiliations.

\section{References}

1. Bray F, Ferlay J, Soerjomataram I, Siegel RL, Torre LA, Jemal A. Global cancer statistics 2018: GLOBOCAN estimates of incidence and mortality worldwide for 36 cancers in 185 countries. CA Cancer J Clin. 2018;68:394-424.

2. Travis WD, Brambilla E, Riely GJ. New pathologic classification of lung cancer: relevance for clinical practice and clinical trials. J Clin Oncol. 2013;31:992-1001.

3. Govindan R, Page N, Morgensztern D, Read W, Tierney R, Vlahiotis A, et al. Changing epidemiology of small-cell lung cancer in the United States over the last 30 years: analysis of the surveillance, epidemiologic, and end results database. J Clin Oncol. 2006;24:4539-44.

4. Gridelli C, Rossi A, Carbone DP, Guarize J, Karachaliou N, Mok $\mathrm{T}$, et al. Non-small-cell lung cancer. Nat Rev Dis Prim. 2015;1:15009.

5. Hershko A, Ciechanover A. The ubiquitin system. Annu Rev Biochem. 1998;67:425-79.

6. Harrigan JA, Jacq X, Martin NM, Jackson SP. Deubiquitylating enzymes and drug discovery: emerging opportunities. Nat Rev Drug Discov. 2018;17:57-78.

7. Hoeller D, Dikic I. Targeting the ubiquitin system in cancer therapy. Nature. 2009;458:438-44.

8. Lipkowitz S, Weissman AM. RINGs of good and evil: RING finger ubiquitin ligases at the crossroads of tumour suppression and oncogenesis. Nat Rev Cancer. 2011;11:629-43.

9. Senft D, Qi J, Ronai ZA. Ubiquitin ligases in oncogenic transformation and cancer therapy. Nat Rev Cancer. 2018; 18:69-88

10. Wertz IE, Wang X. From discovery to bedside: targeting the ubiquitin system. Cell Chem Biol. 2019;26:156-77.

11. Komander D, Clague MJ, Urbe S. Breaking the chains: structure and function of the deubiquitinases. Nat Rev Mol Cell Biol. 2009;10:550-63.

12. Abdul Rehman SA, Kristariyanto YA, Choi SY, Nkosi PJ, Weidlich S, Labib K, et al. MINDY-1 Is a member of an evolutionarily conserved and structurally distinct new family of deubiquitinating enzymes. Mol Cell. 2016;63:146-55.

13. Nijman SM, Luna-Vargas MP, Velds A, Brummelkamp TR, Dirac AM, Sixma TK, et al. A genomic and functional inventory of deubiquitinating enzymes. Cell. 2005;123:773-86.

14. Sacco JJ, Coulson JM, Clague MJ, Urbe S. Emerging roles of deubiquitinases in cancer-associated pathways. IUBMB Life. 2010;62:140-57.

15. Mofers A, Pellegrini P, Linder S, D'Arcy P. Proteasomeassociated deubiquitinases and cancer. Cancer Metastasis Rev. 2017;36:635-53.

16. Song MS, Salmena L, Carracedo A, Egia A, Lo-Coco F, TeruyaFeldstein J, et al. The deubiquitinylation and localization of PTEN are regulated by a HAUSP-PML network. Nature. 2008;455:813-7.

17. Yuan L, Lv Y, Li H, Gao H, Song S, Zhang Y, et al. Deubiquitylase OTUD3 regulates PTEN stability and suppresses tumorigenesis. Nat Cell Biol. 2015;17:1169-81.

18. Zhang J, Zhang P, Wei Y, Piao HL, Wang W, Maddika S, et al. Deubiquitylation and stabilization of PTEN by USP13. Nat Cell Biol. 2013;15:1486-94.

19. Khan OM, Carvalho J, Spencer-Dene B, Mitter R, Frith D, Snijders AP, et al. The deubiquitinase USP9X regulates FBW7 stability and suppresses colorectal cancer. J Clin Investig. 2018;128:1326-37.
20. Kim D, Hong A, Park HI, Shin WH, Yoo L, Jeon SJ, et al. Deubiquitinating enzyme USP22 positively regulates c-Myc stability and tumorigenic activity in mammalian and breast cancer cells. J Cell Physiol. 2017;232:3664-76.

21. Pan J, Deng Q, Jiang C, Wang X, Niu T, Li H, et al. USP37 directly deubiquitinates and stabilizes c-Myc in lung cancer. Oncogene. 2015;34:3957-67.

22. Popov N, Wanzel M, Madiredjo M, Zhang D, Beijersbergen R, Bernards R, et al. The ubiquitin-specific protease USP28 is required for MYC stability. Nat Cell Biol. 2007;9:765-74.

23. Tetreault MP, Yang Y, Katz JP. Kruppel-like factors in cancer. Nat Rev Cancer. 2013;13:701-13.

24. Rowland BD, Peeper DS. KLF4, p21 and context-dependent opposing forces in cancer. Nat Rev Cancer. 2006;6:11-23.

25. Ghaleb AM, Katz JP, Kaestner KH, Du JX, Yang VW. Kruppellike factor 4 exhibits antiapoptotic activity following gammaradiation-induced DNA damage. Oncogene. 2007;26:2365-73.

26. Takahashi K, Yamanaka S. Induction of pluripotent stem cells from mouse embryonic and adult fibroblast cultures by defined factors. Cell. 2006;126:663-76.

27. McConnell BB, Yang VW. Mammalian Kruppel-like factors in health and diseases. Physiol Rev. 2010;90:1337-81.

28. Hu W, Hofstetter WL, Li H, Zhou Y, He Y, Pataer A, et al. Putative tumor-suppressive function of Kruppel-like factor 4 in primary lung carcinoma. Clin Cancer Res. 2009;15:5688-95.

29. Qi XT, Li YL, Zhang YQ, Xu T, Lu B, Fang L, et al. KLF4 functions as an oncogene in promoting cancer stem cell-like characteristics in osteosarcoma cells. Acta Pharm Sin 2019;40:546-55.

30. Chen ZY, Wang X, Zhou Y, Offner G, Tseng CC. Destabilization of Kruppel-like factor 4 protein in response to serum stimulation involves the ubiquitin-proteasome pathway. Cancer Res. 2005;65:10394-400.

31. Hu D, Wan Y. Regulation of Kruppel-like factor 4 by the anaphase promoting complex pathway is involved in TGF-beta signaling. J Biol Chem. 2011;286:6890-901.

32. Kim MO, Kim SH, Cho YY, Nadas J, Jeong CH, Yao K, et al. ERK1 and ERK2 regulate embryonic stem cell self-renewal through phosphorylation of Klf4. Nat Struct Mol Biol. 2012;19:283-90.

33. Zhou H, Liu Y, Zhu R, Ding F, Wan Y, Li Y, et al. FBXO32 suppresses breast cancer tumorigenesis through targeting KLF4 to proteasomal degradation. Oncogene. 2017; 36:3312-21.

34. Hao Z, Sheng Y, Duncan GS, Li WY, Dominguez C, Sylvester J, et al. K48-linked KLF4 ubiquitination by E3 ligase Mule controls T-cell proliferation and cell cycle progression. Nat Commun. 2017;8:14003.

35. Gamper AM, Qiao X, Kim J, Zhang L, DeSimone MC, Rathmell WK, et al. Regulation of KLF4 turnover reveals an unexpected tissue-specific role of pVHL in tumorigenesis. Mol Cell. 2012;45:233-43.

36. Higuchi M, Kawamura H, Matsuki H, Hara T, Takahashi M, Saito $\mathrm{S}$, et al. USP10 Is an essential deubiquitinase for hematopoiesis and inhibits apoptosis of long-term hematopoietic stem cells. Stem Cell Rep. 2016;7:1116-29.

37. Yu T, Chen X, Zhang W, Liu J, Avdiushko R, Napier DL, et al. KLF4 regulates adult lung tumor-initiating cells and represses KRas-mediated lung cancer. Cell Death Differ. 2016;23:207-15.

38. Zhang W, Geiman DE, Shields JM, Dang DT, Mahatan CS, Kaestner KH, et al. The gut-enriched Kruppel-like factor (Kruppel-like factor 4) mediates the transactivating effect of p53 on the p21WAF1/Cip1 promoter. J Biol Chem. 2000;275:18391-8.

39. Yuan J, Luo K, Zhang L, Cheville JC, Lou Z. USP10 regulates p53 localization and stability by deubiquitinating p53. Cell. 2010;140:384-96. 
40. Guturi KKN, Bohgaki M, Bohgaki T, Srikumar T, Ng D, Kumareswaran R, et al. RNF168 and USP10 regulate topoisomerase IIalpha function via opposing effects on its ubiquitylation. Nat Commun. 2016;7:12638.

41. Lim R, Sugino T, Nolte H, Andrade J, Zimmermann B, Shi C, et al. Deubiquitinase USP10 regulates Notch signaling in the endothelium. Science. 2019;364:188-93.

42. Ko A, Han SY, Choi CH, Cho H, Lee MS, Kim SY, et al. Oncogene-induced senescence mediated by c-Myc requires USP10 dependent deubiquitination and stabilization of p14ARF. Cell Death Differ. 2018;25:1050-62.

43. Boulkroun S, Ruffieux-Daidie D, Vitagliano JJ, Poirot O, Charles $\mathrm{RP}$, Lagnaz D, et al. Vasopressin-inducible ubiquitin-specific protease 10 increases ENaC cell surface expression by deubiquitylating and stabilizing sorting nexin 3. Am J Physiol Ren Physiol. 2008;295:F889-900.

44. Zhang M, Hu C, Tong D, Xiang S, Williams K, Bai W, et al. Ubiquitin-specific peptidase 10 (USP10) deubiquitinates and stabilizes MutS homolog 2 (MSH2) to regulate cellular sensitivity to DNA damage. J Biol Chem. 2016;291:10783-91.

45. Bomberger JM, Barnaby RL, Stanton BA. The deubiquitinating enzyme USP10 regulates the post-endocytic sorting of cystic fibrosis transmembrane conductance regulator in airway epithelial cells. J Biol Chem. 2009;284:18778-89.

46. Draker R, Sarcinella E, Cheung P. USP10 deubiquitylates the histone variant H2A.Z and both are required for androgen receptormediated gene activation. Nucleic Acids Res. 2011;39:3529-42.

47. Deng M, Yang X, Qin B, Liu T, Zhang H, Guo W, et al. Deubiquitination and activation of AMPK by USP10. Mol Cell. 2016;61:614-24.

48. Jean JC, George E, Kaestner KH, Brown LA, Spira A, JoyceBrady M. Transcription factor Klf4, induced in the lung by oxygen at birth, regulates perinatal fibroblast and myofibroblast differentiation. PLoS ONE. 2013;8:e54806.

49. Jackson HW, Defamie V, Waterhouse P, Khokha R. TIMPs: versatile extracellular regulators in cancer. Nat Rev Cancer. 2017;17:38-53.

50. Xu C, Hou Z, Zhan P, Zhao W, Chang C, Zou J, et al. EZH2 regulates cancer cell migration through repressing TIMP-3 in nonsmall cell lung cancer. Med Oncol. 2013;30:713.

51. Garofalo M, Di Leva G, Romano G, Nuovo G, Suh SS, Ngankeu A, et al. miR-221\&222 regulate TRAIL resistance and enhance tumorigenicity through PTEN and TIMP3 downregulation. Cancer Cell. 2009;16:498-509. 